Geometry 85 Topology

Volume 5 (2001) 831-883

Published: 21 November 2001

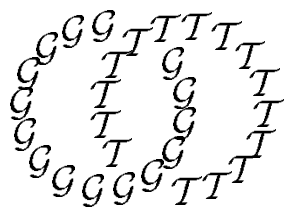

\title{
Concordance and Mutation
}

\author{
P KIRK AND C Livingston \\ Department of Mathematics \\ Indiana University, Bloomington, IN 47405, USA \\ Email: pkirk@indiana.edu, livingst@indiana.edu
}

\begin{abstract}
We provide a framework for studying the interplay between concordance and positive mutation and identify some of the basic structures relating the two.

The fundamental result in understanding knot concordance is the structure theorem proved by Levine: for $n>1$ there is an isomorphism $\phi$ from the concordance group $\mathcal{C}_{n}$ of knotted $(2 n-1)$-spheres in $S^{2 n+1}$ to an algebraically defined group $\mathcal{G}_{ \pm}$; furthermore, $\mathcal{G}_{ \pm}$is isomorphic to the infinite direct sum $\mathbf{Z}^{\infty} \oplus \mathbf{Z}_{2}^{\infty} \oplus \mathbf{Z}_{4}^{\infty}$. It was a startling consequence of the work of Casson and Gordon that in the classical case the kernel of $\phi$ on $\mathcal{C}_{1}$ is infinitely generated. Beyond this, little has been discovered about the pair $\left(\mathcal{C}_{1}, \phi\right)$

In this paper we present a new approach to studying $\mathcal{C}_{1}$ by introducing a group, $\mathcal{M}$, defined as the quotient of the set of knots by the equivalence relation generated by concordance and positive mutation, with group operation induced by connected sum. We prove there is a factorization of $\phi, \mathcal{C}_{1} \stackrel{\phi_{1}}{\longrightarrow} \mathcal{M} \stackrel{\phi_{2}}{\longrightarrow} \mathcal{G}_{-}$. Our main result is that both maps have infinitely generated kernels.

Among geometric constructions on classical knots, the most subtle is positive mutation. Positive mutants are indistinguishable using classical abelian knot invariants as well as by such modern invariants as the Jones, Homfly or Kauffman polynomials. Distinguishing positive mutants up to concordance is a far more difficult problem; only one example has been known until now. The results in this paper provide, among other results, the first infinite families of knots that are distinct from their positive mutants, even up to concordance.
\end{abstract}

AMS Classification numbers Primary: 57M25

Secondary: $57 \mathrm{M} 27$

Keywords: Knot theory, concordance, mutation

Proposed: Ronald Stern

Received: 18 December 2000

Seconded: Robion Kirby, Cameron Gordon

Revised: 17 November 2001

(c) Geometry $8 \mathcal{G}$ Topology $\mathcal{P}$ ublications 


\section{Introduction}

The classical knot concordance group, $\mathcal{C}$, was first defined in [13]. Despite the remarkable progress in low-dimensional topology since then, the structure of this group remains largely a mystery. It is easily shown that $\mathcal{C}$ is countable and abelian. It is a consequence of Levine's work $[33,34]$ that there is a surjective homomorphism $\phi: \mathcal{C} \rightarrow \mathbf{Z}^{\infty} \oplus \mathbf{Z}_{2}^{\infty} \oplus \mathbf{Z}_{4}^{\infty}$. (Throughout this paper $G^{\infty}$ will denote an infinite direct sum of groups and $\mathbf{Z}_{n}$ will denote the finite cyclic group $\mathbf{Z} / n \mathbf{Z}$.) Fox and Milnor [14] noted that $\mathcal{C}$ contains a subgroup isomorphic to $\mathbf{Z}_{2}^{\infty}$; this subgroup is detected by $\phi$. It follows from the work of Casson and Gordon $[5,6,24]$ that the kernel of $\phi$ contains a subgroup isomorphic to $\mathbf{Z}^{\infty}$. Recently, Livingston [38] described a subgroup of the kernel isomorphic to $\mathbf{Z}_{2}^{\infty}$.

Levine proved that the higher-dimensional analog of $\phi$ is an isomorphism and hence provides a classification of higher-dimensional knots up to concordance. Thus the homomorphism $\phi$ is the fundamental object in understanding the difference between classical knot concordance and the higher dimensional theory.

Beyond these results and their purely algebraic consequences, nothing is known concerning the underlying algebraic structure of $\mathcal{C}$ or the pair $(\mathcal{C}, \phi)$.

Advances in geometric topology have offered new insights into the geometric aspects of the knot concordance group. Freedman's work, $[15,16]$, shows that in the topological category all Alexander polynomial one knots are trivial in $\mathcal{C}$. The work of Donaldson [12] and Witten [61] offer new obstructions in the smooth category to a knot being trivial in $\mathcal{C}$, $[8,54]$. Recently, Cochran, Orr, and Teichner, [9], have described a nontrivial filtration of $\mathcal{C}$, providing new obstructions to a knot being trivial in $\mathcal{C}$ in the topological category. Yet, despite the depth of these advances, the underlying structure of $\mathcal{C}$ remains mysterious.

Since the inception of the study of knot concordance, the role and interplay of various 3-dimensional aspects of knotting and the (basically 4-dimensional) study of concordance has been an ongoing theme. The literature is extensive; topics include the relationship of concordance to amphicheirality [11, 20], primeness [30, 38], invertibility [39], hyperbolicity [43], mutation [53], and periodicity $[49,7]$. Of all of these, positive mutation is, even from a purely 3-dimensional perspective, the most subtle.

The difficulty in distinguishing a knot from its positive mutant stems from the fact that positive mutation preserves the $S$-equivalence class of a knot. (This seems to be known, but a proof has not appeared so one will be given in this paper.) As a consequence, all abelian knot invariants - such as the Alexander 
polynomial and module, signatures, and torsion invariants - are incapable of distinguishing a knot from its positive mutant. Furthermore, because the homology of the $n$-fold branched cyclic cover of a knot and its positive mutant are isomorphic as $\mathbf{Z}\left[\mathbf{Z}_{n}\right]$-modules, there is a correspondence between most of the nonabelian representations that are used to distinguish knots when abelian invariants fail. It is also known that more recently discovered knot invariants such as the Jones, Homfly, and Kauffman polynomials also fail to distinguish a knot from its mutant, though colored versions of these invariants are, at times, strong enough to detect mutation [44]. Other invariants that fail to distinguish a knot from its mutant include its hyperbolic volume [53], the eta invariant [42], the hyperbolic torsion [50], certain quantum invariants [52,35] and the CassonWalker invariants of surgery on the knot [59] and of the 2-fold branched cover of the knot [45].

The difficulties of studying the effect of mutation on concordance are even deeper. In particular, since the algebraic concordance class of a knot is determined by its Seifert form, it follows from the result that positive mutants are $S$-equivalent that positive mutants are algebraically concordant. That is, Levine's homomorphism cannot distinguish a knot from a positive mutant. Kearton [26] observed that the examples in [39] demonstrated that negative mutation can change concordance and soon after asked whether the same was true for positive mutation [29]. The question was answered affirmatively in [32], but as of yet the only result concerning positive mutation and concordance is the single example presented in [32] of two knots that are positive mutants but are not concordant. This paper presents infinite classes of such examples.

In this article we provide a framework for studying the interplay between concordance and positive mutation and then we identify some of the basic structures relating the two.

The results are best described in terms of a new group, the Concordance Group of Mutants, $\mathcal{M}$, defined as the quotient of $\mathcal{C}$ obtained via the equivalence relation generated by positive mutation. With the proof that positive mutants are $S$-equivalent it will follow that Levine's homomorphism factors as $\phi_{2} \circ \phi_{1}$, where $\phi_{1}: \mathcal{C} \rightarrow \mathcal{M}$ and $\phi_{2}: \mathcal{M} \rightarrow \mathbf{Z}^{\infty} \oplus \mathbf{Z}_{2}^{\infty} \oplus \mathbf{Z}_{4}^{\infty}$. Our main result is the following:

Theorem 1.1 The kernels of $\phi_{1}$ and $\phi_{2}$ are infinitely generated, containing subgroups isomorphic to $\mathbf{Z}^{\infty}$. The kernel of $\phi_{2}$ contains a subgroup isomorphic to $\mathbf{Z}_{2}^{\infty}$.

The argument is explicit; we construct families of knots in $S^{3}$ which generate appropriate subgroups of ker $\phi_{1}$ and ker $\phi_{2}$. The results are as follows. Let $K_{a}$ 
denote the $a(a+1)$-twisted double of the unknot. The case $a=2$ is drawn in Figure 2.

Theorem 3.13 Let $\left\{a_{i}\right\}_{i=1}^{\infty}$ be an increasing sequence of positive integers so that the sequence $\left\{2 a_{i}+1\right\}_{i=1}^{\infty}$ is a sequence of primes. Then the knots $K_{a_{i}}$ are linearly independent in $\mathcal{M}$ and generate a $\mathbf{Z}^{\infty}$ subgroup of $\operatorname{ker} \phi_{2}: \mathcal{M} \rightarrow$ $\mathbf{Z}^{\infty} \oplus \mathbf{Z}_{2}^{\infty} \oplus \mathbf{Z}_{4}^{\infty}$.

Figure 3 shows an order 2 knot $K_{T}$. The " $T$ " indicates that a knot $T$ is to be tied in one band and $-T$ is to be tied in the other band. Let $K_{0}$ denote the Figure 8 knot.

Corollary 4.2 For the appropriate choice of knots $\left\{T_{i}\right\}_{i=1}^{\infty}$, the knots $K_{T_{i}} \# K_{0}$ generate an infinite 2-torsion subgroup of $\operatorname{ker} \phi_{2}: \mathcal{M} \rightarrow \mathbf{Z}^{\infty} \oplus \mathbf{Z}_{2}^{\infty} \oplus \mathbf{Z}_{4}^{\infty}$.

Figure 4 shows a knot $K$ and two curves $B_{1}$, and $B_{2}^{*}$ in the complement of $K$. Also shown is a 2 -sphere intersecting $K$ in 4 points. Given a knot $J$ let $K_{J}^{*}$ denote the knot obtained by replacing the solid tori neighborhoods of $B_{1}$ and $B_{2}^{*}$ by the exterior of $J$ and $-J$ respectively. We give a recipe for choosing knots $J_{i}$ so that the following theorem holds.

Theorem 5.3 There exists an infinite collection of knots $J_{1}, J_{2}, \ldots$ so that for any choice of integers $n_{1}, n_{2}, \ldots$ with only finitely many of the $n_{i}$ nonzero, the connected sum $\#_{i} n_{i} K_{J_{i}}^{*}$ is not slice, but the knot obtained from $\#_{i} n_{i} K_{J_{i}}^{*}$ by performing a positive mutation on each summand $K_{J_{i}}^{*}$ using the indicated 2-sphere is slice. In particular the kernel of $\phi_{1}: \mathcal{C} \rightarrow \mathcal{M}$ contains a subgroup isomorphic to $\mathbf{Z}^{\infty}$.

The main technique in proving these results is the use of Casson-Gordon invariants. These are sophisticated invariants which depend on an auxiliary vector in a metabolizer for the linking form on branched covers of the knot (a metabolizer is a subspace of the homology of the branched cover on which the linking form vanishes). In order to show a knot is not slice this method requires that one check that every metabolizer contains a vector for which some appropriate Casson-Gordon invariant is non-trivial. Thus much of our work involves a delicate examination of metabolizers. 
Outline This paper is divided into five sections. Following this introduction, Section 2 presents background material. In Section 2.1 we focus on $3-$ dimensional material, especially mutation and the proof that positive mutation preserves the $S$-equivalence class of a knot. In fact, our proof shows that a knot and its positive mutant admit Seifert surfaces with the same Seifert form. In Section 2.2 we review concordance and describe Levine's homomorphism. Casson-Gordon invariants are defined in Section 2.3 and in Section 2.4 we discuss methods for their calculation. We use two types of Casson-Gordon invariants, signatures and discriminants. Discriminants are essential in working with $\phi_{1}$ while signatures are needed to study $\phi_{2}$. We conjecture that in general signature invariants cannot detect elements in the kernel of $\phi_{1}$.

Casson-Gordon invariants were first presented in two different ways: in terms of intersection forms on infinite cyclic covers [5] and in terms of the limiting behavior of the signatures defined via $n$-fold covers [6]. Here both approaches are needed. Since the appearance of $[5,6]$ the approach of $[5]$ has become standard and is almost always simpler and more conceptual. But for the work of Section 3 we find that the approach of [6] offers a much clearer way to study the problem.

In Section 3 we prove that $\phi_{2}$ has an infinitely generated kernel, containing a subgroup isomorphic $\mathbf{Z}^{\infty}$. After summarizing the basic results of the section we describe in Section 3.1 the needed algebra concerning metabolizers of linking forms. In the Section 3.2 we describe the geometry and algebraic topology of the branched covers of mutant knots and in Section 3.3 we give a new tangle addition property for Casson-Gordon invariants (Theorem 3.11). In much of the work on Casson-Gordon invariants one uses Novikov additivity of the signature; to prove the tangle addition theorem one must work in a context in which additivity fails, and we are forced to look carefully at this failure of additivity via the Wall nonadditivity result [60]. In Section 3.4, we present the necessary examples. One step of the proof that these examples are sufficient to show that the kernel of $\phi_{2}$ contains $\mathbf{Z}^{\infty}$ is a lemma computing certain signatures of torus knots. This lemma is proved in Section 3.5.

In Section 4 we show that the kernel of $\phi_{2}$ also contains torsion, in fact it contains a subgroup isomorphic to $\mathbf{Z}_{2}^{\infty}$. That this is the case for $\phi$ was shown in [40]. We briefly review that work here and demonstrate that the infinite set of elements of order 2 studied in [40] remain distinct when included in $\mathcal{M}$.

Section 5 is devoted to proving that the kernel of $\phi_{1}$ contains a subgroup isomorphic to $\mathbf{Z}^{\infty}$. Here a number of challenging issues arise. The proof proceeds by starting with a family of slice knots and showing that their positive mutants 
are not slice. The proof that the knots themselves are slice is nontrivial; it is carried out in Section 5.1. The work of Sections 3 and 4 used signature invariants associated to Casson-Gordon invariants. In this section we use the discriminant invariants instead. Section 5.2 explores the covers of the knots of interest. In Section 5.3 we prove that the kernel of $\phi_{1}$ is nontrivial and in the process develop the needed results concerning the basic Casson-Gordon invariants associated with the examples. Sections 5.4 and 5.5 present the quite delicate analysis of metabolizers of linking forms associated to the knots that is needed to prove a linear independence result. Section 5.6 is devoted to an algebraic lemma.

The results of this paper generalize to the setting of knots in homology spheres. The proofs are essentially identical and the examples we give apply in this more general setting.

The authors wish to thank M Larsen for providing us with the proof of Lemma 5.14 and J Davis for his help with representation theory.

\section{Background: Mutation, concordance and Casson- Gordon invariants}

\subsection{Classical knot theory and mutation}

We will work in the smooth category, though, via [16], all results carry over to the topological locally flat setting. All homology groups are taken with integer coefficients unless noted otherwise. For the basics of knot theory the reader is refered to [51].

In studying concordance, especially when working with such issues as mutation, it is essential that orientation be carefully tracked. To do so, one must begin with a precise definition: a knot consists of a smooth oriented pair $(S, K)$ where $S$ is diffeomorphic to the 3 -sphere, $S^{3}$, and $K$ is diffeomorphic to the 1 -sphere, $S^{1}$. Knots are equivalent if they are oriented diffeomorphic. With this, such notions as connected sum are defined as they usually are for oriented manifolds and pairs.

This notation can become cumbersome and we will use it only when necessary; we will usually follow the standard conventions, writing simply $K$ to denote a knot and viewing knots simply as oriented 1-spheres embedded in the standard (oriented) $S^{3}$. We will also write $-K$ to denote the knot $\left(-S^{3},-K\right)$, the 
"mirror image" of $K$ with orientation reversed. It is this knot that plays the role of inverse in the concordance group.

\section{Mutation}

Let $K$ be a knot in $S^{3}$ and let $S$ be a 2 -sphere embedded in $S^{3}$ meeting $K$ transversely in exactly 4 points. Then $S$ bounds 3 -balls, $B_{1}$ and $B_{2}$, embedded in $S^{3}$, each of which intersects $K$ in two arcs. The pairs $\left(B_{i}, B_{i} \cap K\right)$ are called tangles and we denote them $T_{1}$ and $T_{2}$. Note that $K$ is recovered as the union $T_{1} \cup_{i d} T_{2}$, where $i d$ denotes the identity map on $S$.

Suppose that $\tau$ is an orientation preserving involution of $S$ with fixed point set (which necessarily consists of two points) disjoint from $K \cap S$. Then we can form a new knot as the union $K^{*}=T_{1} \cup_{\tau} T_{2}$. This knot is called a mutant of $K$. In the case that $\tau$ preserves the orientation of $S \cap K$ it is called a positive mutant.

(Though not used in this paper, it is worth noting here that given a knot $K$ and 2 -sphere $S$ as above, the knot that results from positive mutation is independent of the choice of $\tau$ in the sense that any choice of orientation preserving involution which preserves the orientation of $S \cap K$ gives the same result. [53].)

To each knot $K$ one can find a Seifert surface for it, say $F$, and to $F$ along with a choice of basis for $H_{1}(F)$ one can associate a Seifert matrix, say $V$. Trotter [58] defined a notion of $S$-equivalence of Seifert matrices; roughly stated, $S$-equivalence is an equivalence relation generated by change of basis and stabilization of a certain type. A basic results states that any two Seifert matrices for a given knot are $S$-equivalent. Knots are called $S$-equivalent if they have $S$-equivalent Seifert matrices. We now outline a proof of a result that implies that positive mutation preserves the $S$-equivalence class of a knot.

Theorem 2.1 If a knot $K^{*}$ is a positive mutant of $K$, then there are Seifert surfaces $F$ and $F^{*}$ for $K$ and $K^{*}$, respectively, so that, for an appropriate choice of bases, the Seifert matrices are the same. In particular, $K$, and $K^{*}$ are $S$-equivalent.

Proof To set up notation, let $K$ intersect $S$ in points $p_{+}, p_{-}, q_{+}$, and $q_{-}$, where $p_{+}$is joined to $p_{-}$and $q_{+}$is joined to $q_{-}$via oriented $\operatorname{arcs} A_{1}^{1}$ and $A_{2}^{1}$ in $T_{1}=K \cap B_{1}$. Similarly, $p_{+}$is joined to $q_{-}$and $q_{+}$is joined to $p_{-}$via arcs $A_{1}^{2}$ and $A_{2}^{2}$ in $T_{2}=K \cap B_{2}$. 
Fix a pair of $\operatorname{arcs}$ on $S, A_{1}^{3}$ and $A_{2}^{3}$, joining $p_{+}$to $p_{-}$and $q_{+}$to $q_{-}$on $S$ such that $\tau\left(A_{1}^{3}\right)=A_{2}^{3}$. Then the pair of oriented circles $A_{1}^{1} \cup A_{1}^{3}$ and $A_{2}^{1} \cup A_{2}^{3}$ is the oriented boundary of a properly embedded oriented connected surface $F_{1}$ in $B_{1}$. The proof is a standard argument in relative obstruction theory, considering maps of the complement of the $\operatorname{arcs}\left(A_{1}^{1}\right.$ and $\left.A_{2}^{1}\right)$ to $S^{1}$ and letting $F_{1}$ be the preimage of a regular value. Similarly, the union of arcs $A_{1}^{2} \cup A_{2}^{3} \cup A_{2}^{2} \cup A_{1}^{3}$ forms a circle, and this circle is the oriented boundary of a properly embedded oriented connected surface $F_{2}$ in $B_{2}$. The union $F=F_{1} \cup F_{2}$ is an oriented Seifert surface for $K$. Since $\tau$ is orientation preserving and $\tau\left(A_{1}^{3}\right)=A_{2}^{3}, F_{1}$ and $F_{2}$ can similarly be joined to form an oriented Seifert surface $F^{*}$ for $K^{*}$.

Pick oriented bases for $H_{1}\left(F_{1}\right)$ and $H_{1}\left(F_{2}\right)$. A basis for $H_{1}(F)$ can be formed as the union of these, along with one more element that can be represented by a curve on $F$ that intersects $A_{1}^{3}$ and $A_{2}^{3}$ each exactly once.

It is now clear that there will be a corresponding basis for $H_{1}\left(F^{*}\right)$. Furthermore, working directly from the definition of the Seifert matrix, one sees that the corresponding Seifert matrices are identical as follows. The pairings between elements of the basis represented by curves that miss $S$ are clearly unchanged. Let $\alpha$ be a curve representing the extra element of $H_{1}(F)$ that intersects $A_{1}^{3}$ and $A_{2}^{3}$ each exactly once. It is clear that the linking number of $\alpha$ with the positive push-off of any curve missing $S$ is unchanged (here the fact that the mutation is positive is essential). The self-linking of $\alpha$ with its positive push-off is unchanged (regardless of the sign of the mutation).

\subsection{Concordance, mutation, and the Levine homomorphism}

\section{Concordance}

Returning to our more formal notation momentarily, a knot $(S, K)$ is called slice if there is a proper 4 -manifold pair $(B, D)$ with $B$ diffeomorphic to the 4-ball, $B^{4}, D$ diffeomorphic to the 2-disk, $B^{2}$, and with $\partial(B, D)=(S, K)$. Knots $K_{1}$ and $K_{2}$ are called concordant if the connected sum $K_{1} \#-K_{2}$ is slice. Concordance is an equivalence relation and the set of equivalence classes forms a group under the operation induced by connected sum. This group is called the concordance group, denoted $\mathcal{C}$.

An important alternative approach to concordance is the following. Knots $\left(S_{1}, K_{1}\right)$ and $\left(S_{2}, K_{2}\right)$ are called concordant if there is a properly embedded annulus, $A$ (a diffeomorph of $\left.S^{1} \times[0,1]\right)$, in $S^{3} \times[0,1]$ with $\partial\left(S^{3} \times[0,1], A\right)=$ $\left(S_{1}, K_{1}\right) \cup-\left(S_{2}, K_{2}\right)$. In this case $A$ is called a concordance from $\left(S_{1}, K_{1}\right)$ to $\left(S_{2}, K_{2}\right)$. 


\section{The algebraic concordance group and Levine's homomorphism}

The definition of Levine's algebraic concordance group [33] in dimension 3 proceeds as follows. Consider the set of all even dimensional integer matrices $V$ satisfying $\operatorname{det}\left(V-V^{t}\right)=1$. Such a matrix is called metabolic if it is congruent to a matrix having a half dimensional block of zeroes in its upper left hand corner. Matrices $A_{1}$ and $A_{2}$ are called algebraically concordant if the block sum, $A_{1} \oplus-A_{2}$ is metabolic. In particular $S$-equivalent matrices are algebraically concordant. This defines an equivalence relation and the set of equivalence classes forms a group under block sum called the algebraic concordance group, denoted $\mathcal{G}$. Levine proves in [34] that $\mathcal{G} \cong \mathbf{Z}^{\infty} \oplus \mathbf{Z}_{2}^{\infty} \oplus \mathbf{Z}_{4}^{\infty}$.

There is a homomorphism from $\mathcal{C}$ to $\mathcal{G}$, denoted $\phi$, induced by the map that assigns to a knot an associated Seifert matrix. Levine proves that this is surjective. Its analogue in higher dimensions is injective and either surjective or onto an index 2 subgroup. In dimension 3 it is known that the map is onto, and Casson and Gordon proved that it is not injective. In fact, by [24] and [40] the kernel of Levine's homomorphism contains a subgroup isomorphic to $\mathbf{Z}^{\infty} \oplus \mathbf{Z}_{2}^{\infty}$.

\section{The mutation group}

The two relations, concordance and positive mutation, generate an equivalence relation on the set of knots. Denote the set of equivalence classes by $\mathcal{M}$. Thus the two knots, $K_{1}$ and $K_{2}$, represent the same element of $\mathcal{M}$ if a finite sequence of concordances and positive mutations converts one into the other.

It is clear that if $K_{1}$ and $K_{2}$ represent the same class in $\mathcal{M}$ and $J_{1}$ and $J_{2}$ represent the same class in $\mathcal{M}$, then $K_{1} \# J_{1}$ and $K_{2} \# J_{2}$ represent the same class in $\mathcal{M}$. Hence, connected sum induces an operation on $\mathcal{M}$. Clearly, the unknot serves as an identity under this operation and mirror images provide inverses, so $\mathcal{M}$ is a group. It is easily seen that this is quotient of $\mathcal{C}$. Finally, since by Theorem 2.2 mutation does not alter the image of a knot under Levine's homomorphism, we have the following result.

Theorem 2.2 Levine's homomorphism $\phi: \mathcal{C} \rightarrow \mathcal{G}$ factors as the composition of $\phi_{1}: \mathcal{C} \rightarrow \mathcal{M}$ and $\phi_{2}: \mathcal{M} \rightarrow \mathcal{G}$. 


\subsection{Casson-Gordon invariants}

\section{Casson-Gordon invariants as obstructions to slicing}

We now present the formulations of Casson-Gordon invariants that will be needed. Let $K$ be a knot in $S^{3}$ and let $M_{d}$ denote its $d$-fold cyclic branched cover. In what follows $d$ will always be a prime power, $q^{n}$, and in particular, $H_{1}\left(M_{d}\right)$ will always be finite; in fact, $M_{d}$ is a $\mathbf{Z}_{q}$-homology sphere [5]. There is a symmetric linking form on the finite abelian group $H_{1}\left(M_{d} ; \mathbf{Z}\right)$,

$$
l k: H_{1}\left(M_{d} ; \mathbf{Z}\right) \times H_{1}\left(M_{d} ; \mathbf{Z}\right) \rightarrow \mathbf{Q} / \mathbf{Z} .
$$

This linking form is nonsingular in the sense that it defines an isomorphism $H_{1}\left(M_{d} ; \mathbf{Z}\right) \rightarrow \operatorname{Hom}\left(H_{1}\left(M_{d} ; \mathbf{Z}\right), \mathbf{Q} / \mathbf{Z}\right)$.

Definition 2.3 A metabolizer for the linking form on $H_{1}\left(M_{d} ; \mathbf{Z}\right)$ is a subgroup $A \subset H_{1}\left(M_{d} ; \mathbf{Z}\right)$ for which $A=A^{\perp}$. By nonsingularity we have $|A|^{2}=$ $\left|H_{1}\left(M_{d} ; \mathbf{Z}\right)\right|$.

Next, let $p$ be prime power, and let $\chi$ be a character, $\chi: H_{1}\left(M_{d}\right) \rightarrow \mathbf{Z}_{p}$. We will define two invariants in this setting, $\delta(K, \chi)$ and $\sigma_{b}(K, \chi, i)$. (The subscript $b$ stands for bounded, and $i$ is an integer, $0<i<p$. The invariants $\delta$ and $\sigma_{b}$ can be defined for arbitrary prime power covers of $K$, but we will only define and work with $\sigma_{b}$ for the 2 -fold covers, and with $\delta$ for the 3 -fold covers.) The main theorem, derived from the work of Casson and Gordon in $[5,6]$ is the following.

Theorem 2.4 If $K$ is slice there is a metabolizer $A$ of $H_{1}\left(M_{d}\right)$ which is invariant under the action of the group of deck transformations acting on $H_{1}\left(M_{d}\right)$ so that, for any $\chi: H_{1}\left(M_{d}\right) \rightarrow \mathbf{Z}_{p}$ that vanishes on $A$, one has $\sigma_{b}\left(M_{d}, \chi\right)=0$ and $\delta\left(M_{d}, \chi\right)=1$.

Note that $\sigma_{b}\left(M_{d}, \chi\right)$ will be seen to take values in an additive group and $\delta\left(M_{d}, \chi\right)$ will take values in a multiplicative group, and that is why one takes value 0 and the other takes value 1 . The proof of this theorem is contained in the rest of this subsection after presenting the relevant definitions.

\section{Definition of the Casson-Gordon invariant $\sigma_{b}$}

To begin we need to review bordism theory. A good reference for details is [10]. The $n$-dimensional bordism group of a group $G$, denoted $\Omega_{n}(G)$, is defined to be the set of equivalence classes of pairs $\left(M^{n}, \chi\right)$ where $M^{n}$ is a connected 
oriented closed manifold and $\chi$ is a homomorphism from $\pi_{1}\left(M^{n}\right)$ to $G$; two such pairs, $\left(M_{1}^{n}, \chi_{1}\right)$ and $\left(M_{2}^{n}, \chi_{2}\right)$ are considered equivalent if there is an oriented and compact $(n+1)$ manifold $W^{n+1}$ with boundary the disjoint union of $M_{1}^{n}$ and $-M_{2}^{n}$ and a homomorphism from $\pi_{1}\left(W^{n+1}\right)$ to $G$ restricting to give $\chi_{1}$ and $\chi_{2}$ on the boundary. The group structure is induced by the connected sum operation; the group is abelian.

Basic results in bordism theory give that for the trivial group $0, \Omega_{0}(0) \cong \mathbf{Z}$, and $\Omega_{1}(0) \cong \Omega_{2}(0) \cong \Omega_{3}(0) \cong 0$. There is a bordism spectral sequence with $E^{2}$ term given by $H_{i}\left(G, \Omega_{j}(0)\right)$. It follows readily that for $n \leq 3$, the natural map $\Omega_{n}(G) \rightarrow H_{n}(G)$ is an isomorphism. In particular, $\Omega_{3}\left(\mathbf{Z}_{p}\right) \cong \mathbf{Z}_{p}$.

To apply this in our situation, let $\chi_{1}$ be a $\mathbf{Z}_{p}$-valued character defined on $H_{1}\left(M_{2}\right)$. For each integer $k$ there is a natural projection map $H_{1}\left(M_{2^{k}}\right) \rightarrow$ $H_{1}\left(M_{2}\right)$, and hence an induced character $\chi_{k}: H_{1}\left(M_{2^{k}}\right) \rightarrow \mathbf{Z}_{p}$. Thus $p$ copies of $\left(M_{2^{k}}, \chi_{k}\right)$ bound a pair $\left(W_{k}, \chi_{k}\right)$. More precisely, since $\Omega_{3}\left(\mathbf{Z}_{p}\right) \cong \mathbf{Z}_{p}$, $p\left(M_{2^{k}}, \chi_{k}\right)=0$ in $\Omega_{3}\left(\mathbf{Z}_{p}\right)$. This means that there is a 4-manifold and character $\left(W_{k}, \chi_{k}^{\prime}\right)$ whose boundary is $p$ disjoint copies of $M_{2^{k}}$ and with $\chi_{k}^{\prime}$ restricting to $\chi_{k}$ on each boundary component. Let $\tilde{W}_{k}$ denote the corresponding $p$-fold cover, with group of deck transformations isomorphic to $\mathbf{Z}_{p}$, generated by $R_{k}$.

Under the action of $R_{k}$, the homology group $H_{2}\left(\tilde{W}_{k}, \mathbf{C}\right)$ splits into eigenspaces $H_{k, i}=H_{2}\left(\tilde{W}_{k}, \mathbf{C}\right)_{\zeta^{i}}$, where $\zeta$ is a fixed primitive $p$ th root of unity, $1 \leq i \leq$ $p-1$. Let $\sigma(K, \chi, k, i)=\frac{1}{p}\left(\operatorname{signature}\left(H_{k, i}\right)-\operatorname{signature}\left(H_{2}\left(W_{k}, \mathbf{C}\right)\right)\right)$, where the signatures are those of the intersection forms of the 4-manifolds, properly restricted.

At this point one can consider the sequence $\sigma_{b}(K, \chi, i)=\{\sigma(K, \chi, k, i)\}_{k=1, \ldots, \infty}$. Casson and Gordon prove in [5]:

Theorem 2.5 If $K$ is slice, there is a metabolizer $A$ of $H_{1}\left(M_{2} ; \mathbf{Z}\right)$ such that if $\chi$ is a $\mathbf{Z}_{p}$-valued character vanishing on $A$, then for each $i$, the sequence $\{\sigma(K, \chi, k, i)\}_{k}$ is bounded.

As a result, if we view the sequence as in $\left(\prod_{1}^{\infty} \mathbf{Q}\right)_{b}$, the set of all infinite sequences modulo bounded sequences, we have $\sigma_{b}(K, \chi, i)=0 \in\left(\prod_{1}^{\infty} \mathbf{Q}\right)_{b}$ and the $\sigma_{b}$ part of Theorem 2.4 follows.

\section{Definition of the Casson-Gordon invariant $\delta$}

The Casson-Gordon invariant $\delta$ was first defined via a discriminant of a CassonGordon Witt class valued invariant $\tau$ defined in [5]. See [36, 19] for details. 
However, it was shown in [31] that if one is interested only in the discriminant, the 4 -dimensional work of defining $\delta$ can be bypassed and there is a simple 3 -dimensional interpretation in terms of twisted Alexander polynomials.

Let $\bar{M}_{d}$ denote the 3 -manifold obtained from $M_{d}$ by removing the lift of $K$ in the branched cover; that is, $\bar{M}_{d}$ is the $d$-fold cyclic cover of the complement of $K$. There is an inclusion of $H_{1}\left(\bar{M}_{d}\right) \rightarrow H_{1}\left(M_{d}\right)$ and hence an induced character $\chi^{\prime}: H_{1}\left(\bar{M}_{d}\right) \rightarrow \mathbf{Z}_{p}$. Let $\tilde{M}$ denote the infinite cyclic cover of the complement of $K$. It maps to $\bar{M}_{d}$ and hence there is a character $\tilde{\chi}: H_{1}(\tilde{M}) \rightarrow \mathbf{Z}_{p}$.

Suppose that $d$ and $p$ are odd prime powers. Letting $\zeta_{p}$ denote a primitive $p$ th root of unity, one can consider the twisted homology group $H_{1}\left(\tilde{M}, \mathbf{Q}\left(\zeta_{p}\right)\right)$ where the twisting is via $\tilde{\chi}$. Now $\mathbf{Z}$ acts on $\tilde{M}$ via deck transformations, where $\tilde{M}$ is viewed as the infinite cyclic cover of $M_{d}$. Hence the homology group $H_{1}\left(\tilde{M}, \mathbf{Q}\left(\zeta_{p}\right)\right)$ is a $\mathbf{Q}\left(\zeta_{p}\right)\left[t, t^{-1}\right]$ module. This ring is a PID. For the moment let $D$ denote the order of that module (which is a torsion module according to Casson and Gordon [5]). This is an element of $\mathbf{Q}\left(\zeta_{p}\right)\left[t, t^{-1}\right]$, well defined up to units. The discriminant Casson-Gordon invariant $\delta(M, \chi)$ is defined to be the image of $D$ in the the multiplicative group $\mathbf{Q}\left(\zeta_{p}\right)\left[t, t^{-1}\right]^{\times} / N$, where the $\times$ denotes nonzero elements and $N$ denotes the subgroup consisting of norms: elements of the form $f(t) \overline{f(t)}$ where the bar acts by complex conjugation on the coefficients and by sending $t$ to $t^{-1}$.

The theorem proved in [31] from which the second half of Theorem 2.4 follows is the following.

Theorem 2.6 If $K$ is slice, there is a $\mathbf{Z}_{d}$-invariant metabolizer $A$ for $H_{1}\left(M_{d}\right)$ such that for all characters $\chi$ vanishing on $A, \delta\left(M_{d}, \chi\right)=1 \in \mathbf{Q}\left(\zeta_{p}\right)\left[t, t^{-1}\right]^{\times} / N$.

\subsection{Computation of Casson-Gordon invariants}

\section{Additivity of Casson-Gordon invariants}

Given knots $K_{1}$ and $K_{2}$ and characters $\chi_{1}$ and $\chi_{2}$ on the appropriate $d$-fold covers, one has an additivity property for Casson-Gordon invariants. Such a result was first proved in $[24,17]$. In the present context the result follows almost immediately from the definition.

Theorem 2.7 In the setting just described,

$$
\sigma_{b}\left(K_{1} \# K_{2}, \chi_{1} \# \chi_{2}\right)=\sigma_{b}\left(K_{1}, \chi_{1}\right)+\sigma_{b}\left(K_{2}, \chi_{2}\right)
$$

and

$$
\delta\left(K_{1} \# K_{2}, \chi_{1} \# \chi_{2}\right)=\delta\left(K_{1}, \chi_{1}\right) \cdot \delta\left(K_{2}, \chi_{2}\right)
$$




\section{The geometry of satellite knots and their covers}

In general the computation of Casson-Gordon invariants for a particular knot can be quite difficult. However, it is often sufficient to understand how the invariants change under particular geometric operations. Roughly stated, if a geometric construction on a knot yields a slice knot, then it is known that certain Casson-Gordon invariants vanish, so the invariants of the original knot are determined by the effect of the geometric construction. This underlying theme has been central to most calculations since it was first used in $[17,37]$.

The construction that we are most interested in is that of forming a satellite knot from a given knot. The following perspective on this operation was first described in [37].

Let $U$ be an unknot in $S^{3}$ and let $J$ be a knot in $S^{3}$. Removing a tubular neighborhood of $U$ and sewing in the complement of $J$ results in $S^{3}$ again, provided that the meridian and longitude of $J$ are mapped to the longitude and meridian of $U$, respectively. (The resulting space is the union of a knot complement and a solid torus that "fills in" the knot.)

If $K$ is a knot in the complement of $U$, then after this operation $K$ represents a perhaps different knot, $K^{\prime}$, in $S^{3}$. In effect, viewed in the complement of $U$, $K$ represents a knot in a solid torus. The knot $K^{\prime}$ is formed by tying that solid torus into the knot $J$. (The standard terminology is to call $K^{\prime}$ a satellite knot with companion $J$ and embellishment $K$.)

\section{The effect of the satellite operation on $\sigma_{b}$}

For a different form of Casson-Gordon invariants the connection between the invariants and satellite knots was worked out by Litherland [37]. Hence we only present an outline of the approach and give the details as they differ from [37] and are needed here.

We will suppose that $U$ is nullhomologous in the complement of $K$. Hence, the 2-fold branched cover of $K^{\prime}, M_{2}^{\prime}$, is obtained from the the 2-fold branched cover of $K$ by removing the two lifts of $U$ from $M_{2}$ and replacing each with the complement of $J$. This has no effect on the homology, and hence there is a correspondence between $\mathbf{Z}_{p}$-valued characters $\chi$ on $H_{1}\left(M_{2}\right)$ and $\mathbf{Z}_{p}$-valued characters on $H_{1}\left(M_{2}^{\prime}\right)$.

Suppose that $\chi$ takes value $a$ on one lift of $U$. Then it takes value $-a$ on the other lift, since the deck transformation of the 2 -fold cover of a knot acts by multiplication by -1 on the first homology. 
Recall that the (Levine-Tristram) signature function $\sigma_{t}(K), t \in \mathbf{R}$ of a knot $K$ is the signature of the symmetric matrix

$$
B\left(e^{2 \pi i t}\right)=\left(1-e^{2 \pi i t}\right) V+\left(1-e^{-2 \pi i t}\right) V^{T}
$$

where $V$ is a Seifert matrix for $K$. Note that $\sigma_{t}(K)=\sigma_{-t}(K)=\sigma_{t+n}(K)$ for $n \in \mathbf{Z}$, so that $\sigma_{t}(K)$ is determined by its values for $t \in\left[0, \frac{1}{2}\right]$ and also that $\sigma_{t}(K)=-\sigma_{t}(-K)$.

Now, considering the $2^{k}$-fold covers, $M_{2^{k}}^{\prime}$ is obtained from $M_{2^{k}}$ by removing the $2^{k}$ lifts of $U$ and replacing them with copies of the complement of $J$. Under the character on these covers corresponding to $\chi$, half of the lifts of $U$ map to $a$ and half map to $-a$. This motivates the following result. Notice that in the statement of this theorem, the term $\left(2^{k} \sigma_{j a / p}(J)\right)$ represents a sequence in $\left(\prod_{1}^{\infty} \mathbf{Q}\right)_{b}$, the group of infinite sequences modulo bounded sequences.

Theorem 2.8 If $U$ is unknotted and null homologous in the complement of $K$ and $\chi$ and $\chi^{\prime}$ are corresponding characters on the covers $M_{2}$ and $M_{2}^{\prime}$ just described, then $\sigma_{b}(K, \chi, j)-\sigma_{b}\left(K^{\prime}, \chi^{\prime}, j\right)=2^{k} \sigma_{j a / p}(J)$.

Proof The idea of the proof is simple and follows the approach of [37] or [18]. It is actually implicit in [17]. Basically, if $W$ denotes a 4-manifold used in the computation of $\sigma_{b}(K, \chi, j)$ then a 4-manifold that can be used to compute $\sigma_{b}\left(K^{\prime}, \chi^{\prime}, j\right)$ is built from $W$ by adding copies of a 4 -manifold $Y$ along the lifts of $U$. The manifold $Y$ is a 4-manifold with boundary 0 -surgery on $J, N$, for which the natural map of $H_{1}(N) \rightarrow \mathbf{Z}$ extends. But it is this manifold $Y$ that can be used to compute the classical signatures of $J$. Notice that since $\sigma_{j a / p}(J)=\sigma_{-j a / p}(J)$ the sign issue disappears.

\section{The effect of the satellite operation on $\delta$}

In the examples we will be considering in which $\delta$ must be evaluated the situation will be somewhat different. In these cases $U$ will not be null-homologous in $S^{3}-K$; in fact, $U$ will represent $d$ times a generator of $H_{1}\left(S^{3}-K\right)$ and we will be considering the $d$-fold cover of the knot. (We can fix an orientation of $U$ so that $d$ is positive, and use this orientation to orient the lifts of $U$ in what follows.) In this case, $U$ lifts to $d$ curves, say $\left\{U_{i}\right\}_{i=1, \ldots, d}$, where the deck transformation $T$ cyclicly permutes the $U_{i}$.

If we consider a $\mathbf{Z}_{p}$-valued character $\chi$ on $H_{1}\left(M_{d}\right)$ and $\chi$ takes value $a$ on $U_{1}$, then the value of $\chi$ on $U_{i}$ will be $T^{* i}(\chi)(a)$, where $T^{*}$ is the transformation on $H^{1}\left(M_{d} ; \mathbf{Z}_{p}\right)$ induced by $T$. To simplify notation we define $a_{i}=T^{* i}(\chi)(a)$. 
In defining $\delta$ we also needed to consider the representation of $H_{1}\left(\bar{M}_{d}\right)$ to $\mathbf{Z}$. (Recall that $\bar{M}_{d}$ is the cyclic cover of $S^{3}-K$.) In the setting we just describe, it is clear that this representation takes value 1 on each of the $U_{i}$. With this we have:

Theorem 2.9 In the setting just described, $\delta\left(K^{\prime}\right) / \delta(K)=\prod_{i=1}^{d} \Delta_{J}\left(\zeta^{a_{i}} t\right)$, where $\Delta_{J}(t)$ is the Alexander polynomial of $J$.

Proof The idea of the proof is related to that of the previous theorem. In the definition of $\delta$ one considers the infinite cyclic cover of $\bar{M}_{d}$. The infinite cyclic cover of $\bar{M}_{d}^{\prime}$ is built from that of $\bar{M}_{d}$ by removing $d$ copies of $\mathbf{R} \times B^{2}$ and replacing it with $d$-copies of the infinite cyclic cover of $J$. Hence it is expected that $d$ factors of the Alexander polynomial of $J$ should appear. The $\zeta^{a_{i}}$ appear because of the twisting in the homology. That the product appears as it does follows from a Mayer-Vietoris type argument. Details are presented in [30].

\section{The kernel of $\phi_{2}$ contains $\mathbf{Z}^{\infty}$}

We begin with a definition.

Definition 3.1 A knot $K$ is called cg-slice if there exists a metabolizer $A$ for $H_{1}\left(M_{2}\right)$ such that for all prime order characters $\chi$ on $H_{1}\left(M_{2}\right)$ that vanish on $A$, and for all $i, \sigma_{b}(K, \chi, i)=0$.

Hence, the following is a restatement of Theorem 2.5:

Theorem 3.2 If $K$ is slice, then it is $c g$-slice.

Therefore it follows that

Corollary 3.3 If $J$ is concordant to $K$, then $K \#-J$ is cg-slice.

We first prove, in Section 3.1, the following:

Theorem 3.4 If $K_{1}$ is $c g$-slice and $K_{1} \# K_{2}$ is cg-slice, then $K_{2}$ is cg-slice.

The proof is basically algebraic, based on a careful study of metabolizers. The argument is similar to that given by Kervaire [27] in the vector space (as opposed to finite group) setting.

The key result of this section is proved in Section 3.3: 
Theorem 3.5 If $J$ is a positive mutant of $K$ then $K \#-J$ is $c g$-slice.

This result follows from a technical result, a "tangle addition" property for $\sigma_{b}$. As an immediate consequence of these four results we have the corollary that is used to analyze the kernel of $\phi_{2}$ :

Corollary 3.6 If $K$ represents the trivial class in the concordance group of mutants, $\mathcal{M}$, then $K$ is $c g$-slice.

Section 3.4 considers examples. We note first that the original examples in [6] can be used to produce the desired elements in $\operatorname{Ker}\left(\phi_{2}\right)$. We then explore these examples further.

\subsection{Cancellation and metabolizers}

The proof of 3.5 depends an algebraic result, modeled on one presented by Kervaire [27] in the vector space setting.

Let $G_{1}$ and $G_{2}$ be finite abelian groups with linking forms (nonsingular symmetric $\mathbf{Q} / \mathbf{Z}$-valued forms), denoted with brackets: $\langle\rangle:, G_{i} \times G_{i} \rightarrow \mathbf{Q} / \mathbf{Z}$. Suppose that $A_{1}$ is a metabolizer for $G_{1}$ and $A$ is a metabolizer for $G_{1} \times G_{2}$ with respect to the product linking form. Define $A_{2}$ by:

$$
A_{2}=\left\{g \in G_{2} \mid\left(g_{1}, g\right) \in A \text { for some } g_{1} \in A_{1}\right\} .
$$

Theorem $3.7 \quad A_{2}$ is a metabolizer for $G_{2}$.

Proof First note that a subgroup $B$ of $H$, a torsion group with nonsingular linking pairing, is a metabolizer if and only if the linking form vanishes on $B$ and the order of $B$ is the square root of the order of $H$. (From the exact sequence $0 \rightarrow B^{\perp} \rightarrow H \rightarrow \operatorname{Hom}(B, \mathbf{Q} / \mathbf{Z}) \rightarrow 0$ we have that $|B|\left|B^{\perp}\right|=|H|$. If the linking form vanishes on $B$, then $B \subset B^{\perp}$.)

It is clear that the linking form for $G_{2}$ vanishes on $A_{2}$. All that we need to show is that the order of $G_{2}$ is the square of the order of $A_{2}$.

To simplify notation we will view $G_{1}$ and $G_{2}$ as subgroups of $G_{1} \times G_{2}$ in the natural way. Let $p$ denote the projection from $G_{1} \times G_{2}$ to $G_{1}$. Finally, define $A_{0}=\left\{(x, y) \in A \mid x \in A_{1}\right\}$.

We have the two exact sequences:

$$
0 \rightarrow A_{0} \rightarrow A \rightarrow p(A) /\left(A_{0} \cap p(A)\right) \rightarrow 0
$$




$$
0 \rightarrow A_{1} \cap A \rightarrow A_{0} \rightarrow A_{2} \rightarrow 0 .
$$

Notice that $A_{1} \cap A$ pairs trivially against both $p(A)$ and $A_{1}$. Hence it pairs trivially against the sum, $p(A)+A_{1}$. In particular, since the pairing is nonsingular, we have that the orders satisfy $\left|A_{1} \cap A\right|\left|p(A)+A_{1}\right| \leq\left|G_{1}\right|$. Notice also that the order of the sum of groups is equal to the product of their orders, divided by the order of the intersection. Hence

$$
\frac{\left|A_{1} \cap A\right||p(A)|\left|A_{1}\right|}{\left|p(A) \cap A_{1}\right|} \leq\left|G_{1}\right| .
$$

From the second exact sequence we have

$$
\left|A_{2}\right|=\frac{\left|A_{0}\right|}{\left|A_{1} \cap A\right|} .
$$

From the first exact sequence it follows that

$$
\left|A_{2}\right|=\frac{|A|\left|A_{1} \cap p(A)\right|}{\left|A_{0} \cap A\right||p(A)|} .
$$

Using the inequality (along with the fact that $A_{0} \cap p(A)=A_{1} \cap p(A)$ ) applied to the denominator, we have

$$
\left|A_{2}\right| \geq \frac{|A|\left|A_{1}\right|}{\left|G_{1}\right|}
$$

Since each metabolizer has order the square root of the order of its ambient group, and since no self-annihilating subgroup can have order greater than the square root of the order of the ambient group, the result follows.

We can now prove Theorem 3.4.

Proof If $K_{1}$ is cg-slice, let $A_{1}$ be the appropriate metabolizer. Similarly, let $A$ be the metabolizer that exists because $K_{1} \# K_{2}$ is cg-slice. Then the previous theorem provides a metabolizer $A_{2}$. For any $\chi_{2} \in A_{2}$ there is a $\chi_{1} \in A_{1}$ such that $\left(\chi_{1}, \chi_{2}\right) \in A$. Hence, $0=\sigma_{b}\left(K_{1} \# K_{2}\right)=\sigma_{b}\left(K_{1}\right)+\sigma_{b}\left(K_{2}\right)=\sigma_{b}\left(K_{2}\right)$. Hence, $K_{2}$ is cg-slice as desired.

\subsection{Decomposing the branched covers of mutant knots}

\section{The regular cyclic branched cover}

In order to prove a tangle addition theorem for Casson-Gordon invariants in the next section, we must first understand the structure of the relevant covers 
of a knot and its positive mutant. In particular, a detailed understanding of the gluing maps used to assemble the cover as the union of covers of tangles is needed. We develop the necessary results in this section.

If $K$ and $K^{*}$ are positive mutants, let $S$ be the splitting 2-sphere along which the mutation is formed, yielding the tangle decompositions $K=T_{a} \cup_{i d} T_{b}$ and $K^{*}=T_{a} \cup_{\tau} T_{b}$, where $\tau$ is an involution of $S$.

The $2^{k}$-fold cyclic cover of the complement of $K$ corresponds to the kernel of the natural map of $\pi_{1}\left(S^{3}-K\right) \rightarrow \mathbf{Z}_{2^{k}}$. Since $\tau$ preserves the kernel of the corresponding map $\pi_{1}(S-\{S \cap K\}) \rightarrow \mathbf{Z}_{2^{k}}$, and has a fixed point, by elementary covering space theory it lifts to an involution with fixed point on the $2^{k}$-fold branched cover of $S$, denoted $F_{k}$.

Theorem $3.8 \tau_{k}$ acts by multiplication by -1 on $H_{1}\left(F_{k}\right)$.

Proof The quotient of $F_{k}$ under the $\mathbf{Z}_{2}$-action of $\tau_{k}$ is a $2^{k}$-fold branched cover of the quotient of $S$ under the action of $\tau$. This quotient is a 2-sphere and the branch set has quotient consisting of 2 points. The $2^{k}$-fold branched cover of a $S^{2}$ over 2 points is again a 2 -sphere, so the quotient of $F_{k}$ under $\tau_{k}$ is a 2 -sphere.

Since $\tau_{k}$ is an involution, $H_{1}\left(F_{k}, \mathbf{R}\right)$ splits as a direct sum of the +1 and -1 eigenspaces. A transfer argument (see [3]) shows that the +1 eigenspace is isomorphic to the first homology of the quotient, which is trivial since the quotient is a sphere. The theorem follows.

\section{The irregular branched cover}

Since the Casson-Gordon invariants we are interested in are associated with the $p$-fold cyclic covers of the $2^{k}$-fold branched cyclic covers of $K$ and $K^{*}$, we must next consider the decomposition of these spaces.

The $p$-fold cover of $M_{k}$ induced by $\chi$ decomposes as $\tilde{M}_{k}^{a} \cup_{i d} \tilde{M}_{k}^{b}$, where the union is along $\tilde{F}_{k}$, the induced $p$-fold cover of $F_{k}$. Similarly, the $p$-fold cover of $M_{k}^{*}$ induced by $\chi_{k}$ decomposes as $\tilde{M}_{k}^{a} \cup_{\tilde{\tau}_{k}} \tilde{M}_{k}^{b}$ where $\tilde{\tau}_{k}$ is some lift of $\tau_{k}$. Notice that $\tilde{\tau}_{k}$ leaves invariant the preimage of a fixed point of $\tau$ and, by composing with a deck transformation of $\tilde{M}_{k}^{b}$ if need be, we can assume that $\tilde{\tau}_{k}$ fixes a point in that set.

It might be the case that $\tilde{F}_{k}$ is disconnected and this makes the covering space arguments a bit more delicate, since one loses some of the uniqueness properties 
of liftings that exist in the connected case. In particular, for connected covers one has that if a deck transformation fixes a point it is the identity; this is not longer true for disconnected covers.

Theorem 3.9 If $R_{k}$ is the restriction of the order $p$ deck transformation of $\tilde{M}_{k}$ to $\tilde{F}_{k}$, then $\tilde{\tau}_{k}$ and $R_{k}$ together generate a dihedral action on $\tilde{F}_{k}$.

Proof The cover of $M_{k}^{*}$ decomposes as $\tilde{M}_{k}^{a} \cup_{\tilde{\tau}_{k}} \tilde{M}_{k}^{b}$, but note that the cyclic action of the deck transformation restricted to $\tilde{M}_{k}^{b}$ is the inverse of the action that arises when we consider the cover of $M_{k}$, since the character $\chi$ has been replaced by $-\chi$. That the $\mathbf{Z}_{p}$ action is well defined on the union implies that $\tilde{\tau}_{k} \circ R_{k}=R_{k}^{-1} \circ \tilde{\tau}_{k}$.

Consider the restriction of $\tilde{\tau}_{k}$ and $R_{k}$ to the preimage of a fixed point of $\tau_{k}$, a set with $p$ elements. Then $R_{k}$ acts as a $p$-cycle and $\tilde{\tau}_{k}^{2}$ commutes with $R_{k}$. In the symmetric group on $p$ letters the only elements that commute with a $p$-cycle $\rho$ are powers of $\rho$. But $\tilde{\tau}_{k}^{2}$ has a fixed point and the only power of $\rho$ with a fixed point is the identity.

We now have the relations $\tilde{\tau}_{k}^{2}=1, R_{k}^{p}=1$, and $\tilde{\tau}_{k} \circ R_{k}=R_{k}^{-1} \circ \tilde{\tau}_{k}$. Since neither $\tilde{\tau}_{k}$ or $R_{k}$ are trivial, the result follows.

\section{The action on homology}

We now want to understand the action of $\tilde{\tau}_{k}$ and $R_{k}$ on the homology of $\tilde{F}_{k}$. At this point we work with $H_{1}\left(\tilde{F}_{k}, \mathbf{C}\right) \cong H_{1}\left(\tilde{F}_{k}\right) \otimes \mathbf{C}$.

First note that since $R_{k}$ satisfies $R_{k}^{p}=i d$, we have that $H_{1}\left(\tilde{F}_{k}, \mathbf{C}\right)$ splits into $\zeta^{i}$ eigenspaces ( $\zeta$ is a fixed primitive $p$ root of unity). We denote these eigenspaces $H_{\zeta^{i}}, i=0, \ldots, p-1$.

Since $H_{1}\left(\tilde{F}_{k}, \mathbf{C}\right) \cong H_{1}\left(\tilde{F}_{k}\right) \otimes \mathbf{C}$, complex conjugation induces an action on $H_{1}\left(\tilde{F}_{k}, \mathbf{C}\right)$. Via averaging over the action of $R_{k}$, we see that every class in $H_{\zeta^{i}}$ can be represented as a sum of classes of the form $\sum_{j=0}^{p-1} R_{k}^{j} x \otimes \alpha \zeta^{-i j}$. It follows that complex conjugation induces an isomorphism from $H_{\zeta^{i}}$ to $H_{\zeta^{-i}}$. It is also apparent that the intersection forms on these two eigenspaces are conjugate so that the signatures are the same.

Theorem 3.10 For $i \neq 0$ the map on $H_{1}\left(\tilde{F}_{k}, \mathbf{C}\right)$ induced by $\tilde{\tau}_{k}$ maps $H_{\zeta^{i}}$ to $H_{\zeta^{-i}}$ isomorphically, and this isomorphism agrees with the one induced by complex conjugation. 
Proof The action of $\tilde{\tau}_{k}$ and $R_{k}$ generate a dihedral action on the rational vector space $H_{1}\left(\tilde{F}_{k}, \mathbf{Q}\right)$. It follows from representation theory that any such rational representation of the dihedral group splits as the direct sum of three types of representations [55]. The first, denoted simply as $\mathbf{Q}$, is the trivial action on $\mathbf{Q}$. The second, denoted $\mathbf{Q}_{-}$, is the action on $\mathbf{Q}$ for which $R_{k}$ acts trivially and $\tilde{\tau}_{k}$ acts by multiplication by -1 . The last is denoted $\mathbf{Q}[z]$, where $z$ is a primitive $p$ th root of unity, $R_{k}$ acts by multiplication by $z$, and $\tilde{\tau}_{k}$ acts by conjugation, ie, $\tilde{\tau}_{k}\left(z^{i}\right)=z^{p-i}$.

Complexifying (ie, tensoring with $\mathbf{C}$ over $\mathbf{Q}$ ) these representations we see that the only one for which $R_{k}$ has a $\zeta^{i}$ eigenspace is for $\mathbf{Q}[z]$, where that eigenspace is generated by $1+\zeta^{-i} z+\zeta^{-2 i} z^{2}+\ldots+\zeta^{i} z^{p-1}$. Now the action of $\tilde{\tau}_{k}$ is to conjugate; again, this map sends $z^{i}$ to $z^{-i}$. When we complexify the representation, the action of complex conjugation fixes the $z^{i}$ and maps $\zeta^{i}$ to $\zeta^{-i}$. In either case we see the actions are the same. This completes the proof.

\subsection{Tangle addition of Casson-Gordon invariants}

We are now in the following setting. The knots $K$ and $K^{*}$ are positive mutants, split into tangles $T^{a}$ and $T^{b}$ by a splitting sphere $S$. The 2 -fold branched cover of $K, M_{1}$, splits as $M_{1}^{a} \cup_{i d} M_{1}^{b}$ and the 2-fold branched cover of $K^{*}$ splits as $M_{1}^{a} \cup_{\tau_{1}} M_{1}^{b}$, where $\tau_{1}$ is an involution of the 2-fold branched cover of $S, F_{1}$. We have a $\mathbf{Z}_{p}$-valued character $\chi$ on $H_{1}\left(M_{1}\right)$ and a character $\chi^{*}$ on $H_{1}\left(M_{1}^{*}\right)$ obtained from $\chi$ by inverting it on $M_{1}^{b}$. The goal of this section is the proof of the following.

Theorem 3.11 In this setting, $\sigma(K, \chi, k, i)=\sigma\left(K^{*}, \chi^{*}, k, i\right)$.

Proof As expected, the proof begins by decomposing the manifolds used to compute the Casson-Gordon invariants. Hence:

\section{Step 1 Decomposing $M_{k}$}

We begin by describing how to construct a knot $K^{a}$ as the union of $T^{a}$ and a trivial twisted tangle, $T^{c}$, (see Figure 1, illustrating a trivial twisted tangle) so that the restricted character $\chi$ on the 2 -fold branched cover of $T^{a}$ extends to the branched cover of $K^{a}$. There is a representation of $\pi_{1}\left(S^{3}-K\right)$ to the dihedral group, $D_{2 p}=\left\langle t, r \mid t^{2}=1, r^{p}=1, t r t=r^{-1}\right\rangle$, so that its restriction to the index 2 subgroup gives the character $\chi$. (This observation concerning dihedral representations is well known; we present the details in the more complicated 
setting of 3-fold covers in the proof of Theorem 5.4.) The restriction of this representation to $S-\{4$ points $\}$ extends naturally to the complement of some standard twisted tangle, which we denote $T^{c}$.

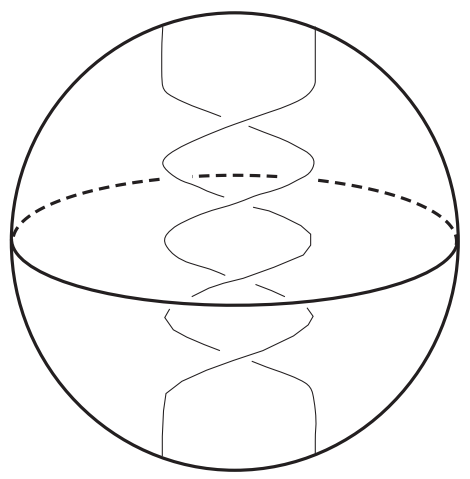

Figure 1: A trivial twisted tangle

Notice that the same tangle $T^{c}$ can be glued to the tangle $T^{b}$ to yield the knot $K^{b}$ for which the character also extends in the same way over the cover of $T^{c}$. We let $M_{k}^{a}, M_{k}^{b}$, and $M_{k}^{c}$ be the induced branched covers of $T^{a}, T^{b}$, and $T^{c}$. Notice that $\tau$ extends to an involution of $T^{c}$, and lifts to give involutions $M_{k}^{c}$.

We can form $N_{k}^{a}=M_{k}^{a} \cup_{i d} M_{k}^{c}$, the $2^{k}$-fold branched cover of $K^{a}$. Similarly, form $N_{k}^{b}=M_{k}^{b} \cup_{i d} M_{k}^{c}$, the $2^{k}$-fold branched cover of $K^{b}$. Let $\chi_{k}^{a} \in H^{1}\left(N_{k}^{a} ; \mathbf{Z}_{p}\right)$ and $\chi_{k}^{b} \in H^{1}\left(N_{k}^{b} ; \mathbf{Z}_{p}\right)$ be the induced characters.

\section{Step 2 Non-additivity of the signature}

We now want to relate the Casson-Gordon invariants of both $N_{k}^{a}$ and $N_{k}^{b}$ to those of $M_{k}$ and $M_{k}^{*}$. As usual, we let $p$ copies of $N_{k}^{a}$ and $N_{k}^{b}$ bound $4^{-}$ manifolds $W_{k}^{a}$ and $W_{k}^{b}$ over $\chi_{k}^{a}$ and $\chi_{k}^{a}$, respectively. The desired manifolds $W_{k}$ and $W_{k}^{*}$ needed to compute the Casson-Gordon invariants for $M_{k}$ and $M_{k}^{*}$ are obtained by gluing $W_{k}^{a}$ and $W_{k}^{b}$ along $M_{k}^{c}$ using either the identity map or the extension of $\tau_{k}$ to $M_{k}^{c}$. We then want to apply additivity of the signature, both on the level of the base manifold and for the eigenspace signature on the $p$-fold cyclic covers. However signatures do not add in this setting; the necessary result is Wall's "non-additivity" formula for signature [60], which we now summarize.

Suppose that a 4 -manifold $Z$ is formed as the union of $Z_{1}$ and $Z_{2}$ along a 3-dimensional submanifold $Y$ contained in both boundaries and the boundary 
of $Y$ is the surface $X$. Then

$$
\operatorname{sign}(Z)=\operatorname{sign}\left(Z_{1}\right)+\operatorname{sign}\left(Z_{2}\right)-\psi\left(H_{1}(X), V_{1}, V_{2}, V_{3}\right),
$$

where $\psi$ is a function defined by Wall and $V_{1}, V_{2}$, and $V_{3}$ are the kernels in $H_{1}(X)$ of the inclusion of $X$ into $\partial Z_{1}-Y, \partial Z_{2}-Y$, and $Y$ respectively.

\section{Step 3 Applying non-additivity}

From this most steps of the theorem follow readily. Using the notation of Section 2, we have

$$
\begin{gathered}
\operatorname{sign}\left(W_{k}\right)=\operatorname{sign}\left(W_{k}^{a}\right)+\operatorname{sign}\left(W_{k}^{b}\right)-\psi\left(H_{1}(F), V_{1}, V_{2}, V_{3}\right) \\
\operatorname{sign}\left(W_{k}^{*}\right)=\operatorname{sign}\left(W_{k}^{a *}\right)+\operatorname{sign}\left(W_{k}^{b^{*}}\right)-\psi\left(H_{1}\left(F^{*}\right), V_{1}^{*}, V_{2}^{*}, V_{3}^{*}\right) .
\end{gathered}
$$

Now, by identifying $F$ (and $F^{*}$ ) with the boundary of $M_{k}^{a}$, we see that all these terms are in fact identical, except perhaps the terms $V_{2}$ and $V_{2}^{*}$. The first is the kernel of $H_{1}(F) \rightarrow H_{1}\left(M_{k}^{b}\right)$ and the second is the kernel of this same inclusion, precomposed with $\tau_{k}$. But since $\tau_{k}$ acts by multiplication by -1 , these are the same as well.

On the eigenspace level the situation is more difficult. First, one needs to observe that Wall's theorem applies on the level of eigenspaces. At the conclusion of [60], Wall notes that the results of his paper hold in the setting of $G$-manifolds and for the $G$-signature. Specifically,

$$
\sigma_{g}\left(W_{k}\right)=\sigma_{g}\left(W_{k}^{a}\right)+\sigma_{g}\left(W_{k}^{b}\right)-\psi_{g}\left(H_{1}(\tilde{F}), V_{1}, V_{2}, V_{3}\right),
$$

where $\sigma_{g}$ denotes the $G$-signature and $\psi_{g}$ is an invariant depending only on the subspaces $V_{i}$ of $H_{1}(\tilde{F})$. Since the eigenspace signatures and the $G$-signatures are equivalent via a Fourier transform by $[5,2]$, it follows that (again using the notation of Section 2, so, for example, $H_{k, i}^{a}$ means the $\zeta^{i}$ eigenspace of $\left.\mathrm{H}_{2}\left(\tilde{W}_{k}^{a}, \mathbf{C}\right)\right)$ we have

$$
\begin{gathered}
\operatorname{sign}\left(H_{k, i}\right)=\operatorname{sign}\left(H_{k, i}^{a}\right)+\operatorname{sign}\left(H_{k, i}^{b}\right)-\psi\left(H_{1}(\tilde{F})_{\zeta^{i}}, V_{1}, V_{2}, V_{3}\right) \\
\operatorname{sign}\left(H_{k, i}{ }^{*}\right)=\operatorname{sign}\left(H_{k, i}^{a}{ }^{*}\right)+\operatorname{sign}\left(H_{k, i}^{b}{ }^{*}\right)-\psi\left(H_{1}(\tilde{F})_{\zeta^{i}}{ }^{*}, V_{1}^{*}, V_{2}^{*}, V_{3}^{*}\right),
\end{gathered}
$$

where $\psi$ is a function depending only on the subspaces $V_{i}$ of $H_{1}(\tilde{F})_{\zeta^{i}}$.

Alternatively, one can check line-by-line that Wall's arguments go through in the setting of eigenspaces and that hence these formulas hold.

By identifying $F_{k}$ with the boundary of $M_{k}^{a}$, we have that all the terms in the sum are identical, except for two pairs that we now deal with. 
Step $4 \operatorname{sign}\left(H_{k, i}^{b}\right)=\operatorname{sign}\left(H_{k, i}^{b}{ }^{*}\right)$

These might be different because the second term is obtained after replacing $\chi_{k}$ with $-\chi_{k}$ on $M_{k}^{b}$. This has the effect of inverting the $\mathbf{Z}_{p}$ action on $\tilde{W}_{k}^{b}$, and thus interchanges the eigenspaces. However, complex conjugation also induces an interchange of eigenspaces, and as we noted in the previous section, this preserves signatures, so the terms are in fact equal.

Step $5 \quad V_{2}=V_{2}^{*}$

This is the most difficult and delicate step. We must prove that the action of the lift of $\tau_{k}, \tilde{\tau}_{k}$, to the $p$-fold cyclic cover preserves the kernels of the inclusions on the eigenspaces. More precisely, what must be shown is that the kernel of $\tilde{i}: H_{1}\left(\tilde{F}_{k}, \mathbf{C}\right)_{\zeta^{i}} \rightarrow H_{1}\left(\tilde{M}_{k}^{b}, \mathbf{C}\right)_{\zeta^{i}}$ and the kernel of $\tilde{i} \circ \tilde{\tau}_{k}: H_{1}\left(\tilde{F}_{k}, \mathbf{C}\right)_{\zeta^{i}} \rightarrow$ $H_{1}\left(\tilde{M}_{k}^{b}, \mathbf{C}\right)_{\zeta^{i}}^{*}$ are the same. Here the subscript $\zeta^{i}$ denotes the $\zeta^{i}$ eigenspace.

As mentioned earlier, there is a natural isomorphism from $H_{1}\left(\tilde{M}_{k}^{b}, \mathbf{C}\right)_{\zeta^{i}}$ to $H_{1}\left(\tilde{M}_{k}^{b}, \mathbf{C}\right)_{\zeta^{-i}}$ induced by complex conjugation and this second eigenspace is isomorphic to $H_{1}\left(\tilde{M}_{k}^{b}, \mathbf{C}\right)_{\zeta^{i}}^{*}$ since the ${ }^{*}$ denotes the groups associated with $-\chi_{k}$ for which the action of the deck transformation is inverted. Also, notice that complex conjugation preserves the kernel of inclusions, since the complex kernel is simply the real kernel tensored with $\mathbf{C}$.

In the last section we proved that $\tilde{\tau}_{k}$ induces an isomorphism of $H_{1}\left(\tilde{F}_{k}, \mathbf{C}\right)_{\zeta^{i}}$ to $H_{1}\left(\tilde{F}_{k}, \mathbf{C}\right)_{\zeta^{-i}}$ that agrees with the one induced by complex conjugation. Theorem 3.11 follows.

Proof of Theorem 3.5 Since $K \#-K$ is slice, Theorem 3.2 says that $K \#-K$ is cg-slice. But, $K \#-J$ is a positive mutant of $K \#-K$. Hence the following theorem completes the proof.

Theorem 3.12 If $K$ is cg-slice and $K^{*}$ is a positive mutant of $K$, then $K^{*}$ is also $c g$-slice.

Proof We have already seen that there is a correspondence between characters on $H_{1}(M)$ and $H_{1}\left(M^{*}\right)$ and that, by Theorem 2.7, $\sigma_{i}$ is invariant under this correspondence. It remains to check that metabolizers are preserved under the correspondence. To see this we need to be look a little more closely at the homology and the linking form.

Via a Mayer-Vietoris argument,

$$
H_{1}(M)=\left(H_{1}\left(M^{a}\right) \oplus H_{1}\left(M^{b}\right)\right) / G_{1}
$$


and

$$
H_{1}\left(M^{*}\right)=\left(H_{1}\left(M^{a}\right) \oplus H_{1}\left(M^{b}\right)\right) / G_{2},
$$

where $G_{1}$ is the subgroup generated by elements of the form $\left(i_{*}(x), i_{*}(x)\right), x \in$ $H_{1}(F)$, and $G_{2}$ is the subgroup generated by the set of elements of the form $\left(i_{*}(x),-i_{*}(x)\right), x \in H_{1}(F)$. An isomorphism is given by the map $(x, y) \rightarrow$ $(x,-y)$.

To check that the isomorphism is an isometry of linking forms, we need only to check that the linking number of classes of the form $(x, 0)$ or $(0, y)$ is preserved.

Recall that the linking form $l k(a, b)$ is defined geometrically by letting a multiple of $a$ bound a chain and computing the intersection number $b$ with that chain, and then dividing by the order of the multiple.

To check that the linking number of a class of the form $(x, 0)$ and a class of the form $(0, y)$ is preserved, let $x$ bound a chain $z$ in $M$. We can assume that $z$ is transverse to $F$ and intersects $F$ in a $\tau$ invariant 1 -chain. It follows that $z$ can then be cut and reglued to give a chain $z *$ in $M^{*}$. Notice though that since $\tau$ acts by -1 on $H_{1}(F)$, the portion of $z *$ in $M^{b}$ has its orientation reversed in the construction. Hence, the intersection number of $z$ with $(0, y)$ in $M$ is the same as the intersection number of $z *$ with $(0,-y)$ in $M *$.

A similar argument applies to the cases of linking numbers of classes of the form $(x, 0)$ and $\left(x^{\prime}, 0\right)$ or of classes of the form $(0, y)$ and $\left(0, y^{\prime}\right)$.

Since the isomorphism of $H_{1}(M)$ with $H_{1}\left(M^{*}\right)$ preserves linking, it takes metabolizers to metabolizers.

\subsection{Examples}

Let $K_{a}$ be the $a(a+1)$ twisted double of the unknot, illustrated in Figure 2 below.

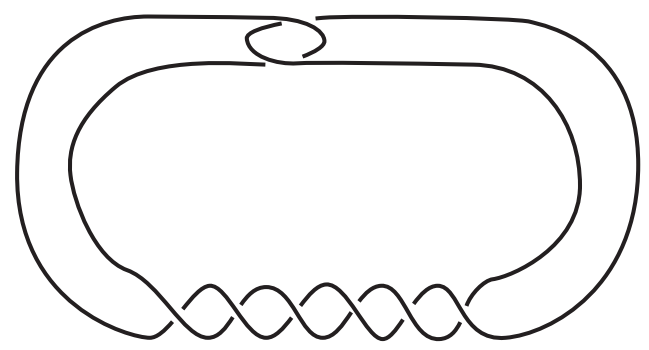

Figure 2: A twisted double of the unknot 
These knots were principal examples in both $[5,6]$ and [24]. In particular, it is shown in $[5,6]$ that for $a>1$ these knots are algebraically slice but not slice. In [24] it is further shown that if a sequence of values of $a>1$ are chosen so that the corresponding sequence of values $2 a+1$ are distinct primes then the associated sequence of knots forms a linearly independent set in the kernel of Levine's homomorphism.

The proofs of the results of the preceding paragraph depend (in the language developed here) on an estimate for $\sigma_{b}$. A careful examination shows that the results in $[5,6,24]$ imply, via Theorem 3.5 , that the sequence is independent in the kernel of $\phi_{2}$. In fact one has the following theorem.

Theorem 3.13 Let $\left\{a_{i}\right\}_{i=1}^{\infty}$ be an increasing sequence of positive integers so that the sequence $\left\{2 a_{i}+1\right\}_{i=1}^{\infty}$ is a sequence of primes. Then the knots $K_{a_{i}}$ are linearly independent in $\mathcal{M}$ and generate a $\mathbf{Z}^{\infty}$ subgroup of $\operatorname{ker} \phi_{2}: \mathcal{M} \rightarrow \mathcal{G}$.

Proof The Seifert form for $K_{a}$ is given by

$$
\left(\begin{array}{cc}
-1 & 1 \\
0 & a(a+1)
\end{array}\right) \text {. }
$$

A symplectic basis for the form is given by the pair of elements $\{(a,-1),(1,0)\}$. In this basis the Seifert form has a 0 in the upper left-hand entry, ie, the vector $(a,-1)$ is a metabolizing vector for the Seifert form. In particular $K_{a}$ is algebraically slice, and hence lies in the kernel of $\phi$; its image in $\mathcal{M}$ lies in the kernel of $\phi_{2}$. By Corollary 3.6 $K_{a}$ is non-trivial in $\mathcal{M}$ if $K_{a}$ is not cg-slice.

Representing the elements $(a,-1)$ and $(1,0)$ by curves that meet once on the Seifert surface, and taking a neighborhood of the pair, one can describe the given Seifert surface as a disk with two bands added, one with core the curve representing $(a,-1)$ and the other with core representing the element $(1,0)$. One can easily check that the first band is tied in a $(-a, a+1)$-torus knot, which we will denote for now by $T_{-a}$. Finally note that tying a copy of $T_{a}$ in the band yields a slice knot, since it now bounds a genus one Seifert surface for which one band has 0 framing and has a core that is slice. We let $K_{a}^{s}$ denote this slice knot.

The 2-fold branched cover of $K_{a}$ (and $\left.K_{a}^{s}\right)$ has homology $\mathbf{Z}_{(2 a+1)^{2}}$. If $(2 a+1)$ is prime, there is a unique metabolizer for this form, and hence a unique (up to multiple) character vanishing on that metabolizer. By Theorem 2.8, tying the knot in the band changes the value of $\sigma_{b}$ by the sequence $\left(2^{k} \sigma_{j /(2 a+1)}\left(T_{a}\right)\right)_{k=1 \ldots \infty}$. In other words, for a character $\chi$ in the metabolizer,

$$
\sigma_{b}\left(K_{a}, \chi, \ell\right)-\sigma_{b}\left(K_{a}^{s}, \chi, \ell\right)=\left(2^{k} \sigma_{j /(2 a+1)}\left(T_{a}\right)\right)_{k=1 \ldots \infty}
$$


for some $j$. The number $j$ is determined by the value of $\chi$ on the curve refered to as $U$ in the statement of Theorem 2.8 .

A careful analysis of the branched cover of $K_{a}^{s}$ shows that $j$ will be nonzero modulo $2 a+1$. Details of such a calculation are presented in [17] or [18]. They are based on the presentation of the 2 -fold cover of $K_{a}^{s}$ using the methods developed in [1].

This result must be 0 in the group of sequence modulo bounded sequences since $K_{a}^{s}$ is slice and since the metabolizer is unique. Therefore the knot $K_{a}$ will have $\sigma_{b}\left(K_{a}, \chi, \ell\right)=\left(2^{k} \sigma_{j /(2 a+1)}\left(T_{a}\right)\right)_{k=1 \ldots \infty}$.

A proof of the following lemma is given in the next section.

Lemma 3.14 Let $K$ denote the $(-a, a+1)$-torus knot with $a>1$. Then the signature function $\sigma_{t}(K)$ is positive for $\frac{1}{a(a+1)}<t<1-\frac{1}{a(a+1)}$.

Since $j$ is non-zero modulo $2 a+1$, we may assume $0<j<2 a+1$, so that

$$
\frac{1}{a(a+1)}<\frac{j}{2 a+1}<1-\frac{1}{a(a+1)}
$$

Therefore Lemma 3.14 implies that $\sigma_{j /(2 a+1)}\left(T_{a}\right)$ is positive and so in particular $K_{a}$ is not cg-slice. Thus Corollary 3.6 implies that $K_{a}$ is non-trivial in $\mathcal{M}$, and hence $K_{a}$ is a non-trivial knot in the kernel of $\phi_{2}$.

To construct the infinite family of linearly independent algebraically slice knots in $\mathcal{M}$, let $\left\{a_{i}\right\}_{i=1}^{\infty}$ be an increasing sequence of positive integers so that the sequence $\left\{2 a_{i}+1\right\}_{i=1}^{\infty}$ is a sequence of distinct primes. We will show the sequence $\left\{K_{a_{i}}\right\}$ is linearly independent. Given a sequence of integers $\left\{n_{i}\right\}$ we must show that the knot $L=\#_{i} n_{i} K_{a_{i}}$ is not slice. By reindexing, and replacing $L$ with $-L$ if necessary, we may assume that $n_{1}$ is positive. We will show that for any character $\chi$ on the 2 -fold branched cover of $L$ taking values in $\mathbf{Z}_{2 a_{1}+1}$ the value of $\sigma_{b}(L, \chi, \ell)$ is nontrivial for any $\ell$.

Since the 2 -fold branched cover of $L$ is a connected sum, we can write $\chi$ as a sum $\#_{i} \chi_{i}$ where $\chi_{i}$ is the restriction of $\chi$ to the cover of $n_{i} K_{i}$. But since the 2 -fold branched cover of $K_{i}$ has no $2 a_{1}+1$ homology for $i>1$, we have that $\chi_{i}$ is trivial for $i>1$ and therefore $\sigma_{b}\left(n_{i} K_{a_{i}}, \chi_{i}, \ell\right)=0$ for $i>1$. Hence by the additivity of $\sigma_{b}$,

$$
\sigma_{b}(L, \chi, \ell)=\sigma_{b}\left(n_{1} K_{a_{1}}, \chi_{1}, \ell\right) .
$$

We will show that $\sigma_{b}\left(n_{1} K_{a_{1}}, \chi_{1}, \ell\right)$ is nonzero. Using additivity again this is the sum of values of $\sigma_{b}\left(K_{a_{1}}, \eta_{m}, \ell\right)$ where $\eta_{m}$ denotes the restriction of $\chi_{1}$ to 
the $m$ th factor of $n_{1} K_{a_{1}}=K_{a_{1}} \# K_{a_{1}} \# \cdots \# K_{a_{1}}$. Explicitly

$$
\sigma_{b}\left(n_{1} K_{a_{1}}, \chi_{1}, \ell\right)=\sum_{m=1}^{n_{1}} \sigma_{b}\left(K_{a_{1}}, \eta_{m}, \ell\right)=\sum_{i=m}^{n_{1}}\left(2^{k} \sigma_{j_{m} /\left(2 a_{1}+1\right)}\left(T_{a_{1}}\right)\right)_{k=1, \ldots, \infty} .
$$

Since $\chi$ is nontrivial, at least one of the $\eta_{m}$ is nontrivial. For each such $m$, $j_{m}$ is non-zero modulo $2 a_{1}+1$ as before, and Lemma 3.14 implies that the corresponding $\sigma_{j_{m} /\left(2 a_{1}+1\right)}\left(T_{a_{1}}\right)$ is positive.

For those $m$ such that $\eta_{m}$ is trivial, $\sigma_{j_{m} /\left(2 a_{1}+1\right)}\left(T_{a_{1}}\right)$ is zero. Therefore, $\sigma_{b}\left(n_{1} K_{a_{1}}, \chi_{1}, \ell\right)$ is non-zero. Since $\sigma_{b}\left(n_{1} K_{a_{1}}, \chi_{1}, \ell\right)=\sigma_{b}(L, \chi, \ell), L$ is not cgslice and so represents a non-trivial element in $\mathcal{M}$ by Corollary 3.6. This concludes the proof of Theorem 3.13.

\subsection{The Proof of Lemma 3.14}

The proof of this result concerning the signatures of $(a, a+1)$-torus knots could presumably be constructed using a detailed analysis of the Seifert form of these knots. See [47] for an example of such a computation, carried out in the case of $1 / 2$ signatures in detail. However, the recently developed understanding of the signatures in terms of $S U(2)$ representations offers an alternative which, though more sophisticated in ways, brings a simple perspective.

Proof of Lemma 3.14 We begin by recalling that $S U(2)$ can be identified with the unit quaternions via

$$
\left(\begin{array}{cc}
z & w \\
-\bar{w} & \bar{z}
\end{array}\right) \leftrightarrow z+w \mathbf{j}
$$

for $z, w \in \mathbf{C},|z|^{2}+|w|^{2}=1$.

Results of Herald [21] (see also [23]) interpret the signature function of a knot in terms of the space of conjugacy classes of $S U(2)$ representations of the fundamental group of the knot complement and the trace of these representations restricted to the meridian, $\mu$, of the knot. Roughly stated, $\sigma_{t}$ is equal to twice the algebraic count of conjugacy classes of non-abelian $S U(2)$ representations $\rho$ of the knot group satisfying $\operatorname{tr}(\rho(\mu))=2 \cos (\pi t)$. In general one must perturb the representation space to assure transversality before performing the count, but it can be shown that for $(p, q)$-torus knots such a perturbation is unnecessary. Furthermore, in general there is a sign issue in performing the count, but it follows from the work of Herald [22] that for a $(p, q)$-torus knot each representation contributes $-\operatorname{sign}(p q)$. 
It follows from the previous discussion that a proof of Lemma 3.14 is implied by the statement that for all $t, \frac{1}{a(a+1)}<t<1-\frac{1}{a(a+1)}$, there is a nonabelian $S U(2)$ representation, $\rho$, of the $(-a, a+1)$-torus knot group with $\operatorname{tr}(\rho(\mu))=2 \cos (\pi t)$. The rest of this proof is devoted to proving the existence of such a representation.

Recall that $\pi=\pi_{1}\left(S^{3}-K\right)=\left\langle x, y \mid x^{a}=y^{a+1}\right\rangle$ and that a simple calculation shows that the meridian is represented by $\mu=x y^{-1}$.

Given a pair $m, n$ of integers such that $0<m<a, 0<n<a+1$ with $m \equiv n$ $(\bmod 2)$, and $u \in[0,1]$, there is a representation $\rho_{u, m, n}: \pi \rightarrow S U(2)$ satisfying

$$
\rho_{u, m, n}(x)=e^{2 \pi i m / 2 a}
$$

and

$$
\rho_{u, m, n}(y)=\cos (2 \pi n / 2(a+1))+\sin (2 \pi n / 2(a+1))(\cos (\pi u) \mathbf{i}+\sin (\pi u) \mathbf{j}) .
$$

(These two unit quaternions are easily shown to be $a$ and $a+1$ roots of 1 or -1 , depending on whether $m$ and $n$ are even or odd.) The representation $\rho_{u, m, n}$ is nonabelian unless $u=0$ or 1 .

Setting $u=0$ and 1 gives

$$
\rho_{0, m, n}(\mu)=\exp \left(2 \pi i\left(\frac{m}{2 a}-\frac{n}{2(a+1)}\right)\right)
$$

and

$$
\rho_{1, m, n}(\mu)=\exp \left(2 \pi i\left(\frac{m}{2 a}+\frac{n}{2(a+1)}\right)\right) .
$$

The traces of these values, given as twice the real parts of the unit quaternions, are $2 \cos \left(\pi\left(\frac{m}{a}-\frac{n}{(a+1)}\right)\right)$ and $2 \cos \left(\pi\left(\frac{m}{a}+\frac{n}{(a+1)}\right)\right)$. By continuity (in $u$ ) all traces between these two values occur also.

That the corresponding open intervals cover $\left(\frac{1}{a(a+1)}, 1-\frac{1}{a(a+1)}\right)$ can be seen just by considering the pairs $(m, n)=(1,2 k+1), k=0,1,2, \cdots$.

\section{The kernel of $\phi_{2}$ contains $\mathrm{Z}_{2}^{\infty}$}

In [40] it was shown that the kernel of Levine's homomorphism contains a subgroup isomorphic to $\mathbf{Z}_{2}^{\infty}$. Here we will show that those examples remain nontrivial in $\mathcal{M}$ and that the kernel of $\phi_{2}$ contains an infinite collection of elements of order 2. The proof is much like that of [40] and we will only outline the arguments, highlighting the points where they have to be enhanced. 
Let $K_{T}$ be the knot illustrated in Figure 3. The figure illustrates a genus 1 Seifert surface for $K_{T}$ in which one band has the knot $T$ tied in it and the other has $-T$ tied in it. The bands are twisted so that the Seifert form is

$$
\left(\begin{array}{cc}
1 & 1 \\
0 & -1
\end{array}\right)
$$

In the case that $T$ is the unknot $K_{T}$ is the Figure 8 knot, $K_{0}$. In general it is easily seen that $K_{T}=-K_{T}$ and hence that $K_{T}$ is of order 1 or 2 in $\mathcal{C}$. (Fox and Milnor were the first to show that the order of $K_{0}$ is exactly 2 , using the Alexander polynomial.)

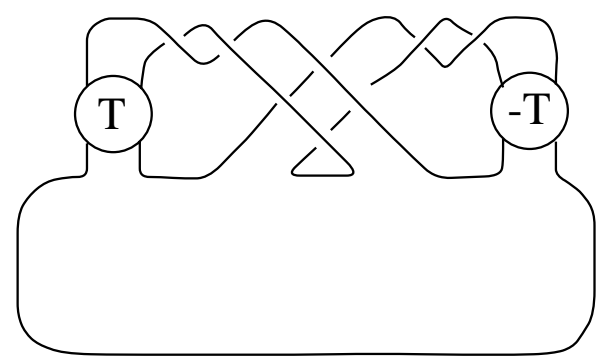

Figure 3: An order 2 knot

The examples we want to consider are knots of the form $K_{T} \# K_{0}$, for particular knots $T$. Note immediately that these knots are of order either 1 or 2 in $\mathcal{M}$. Also, they are in the kernel of $\phi_{2}$, since the two summands have the same Seifert form, the Seifert form of an order 2 knot. We will show that for an appropriate set of knots $\left\{T_{i}\right\}_{i=1}^{\infty}$, the knots $K_{T_{i}} \# K_{0}$ are distinct in $\mathcal{M}$. Hence, the kernel of $\phi_{2}: \mathcal{M} \rightarrow \mathcal{G}$ contains $\mathbf{Z}_{2}^{\infty}$.

Showing that $K_{T_{i}} \# K_{0} \neq K_{T_{j}} \# K_{0}$ in $\mathcal{M}$ is clearly equivalent to showing that $K_{T_{i}} \# K_{0} \# K_{T_{j}} \# K_{0}$ is non zero in $\mathcal{M}$. But this connected sum is the same (up to concordance) as $K_{T_{i}} \# K_{T_{j}}$, since the $K_{0}$ summands cancel.

Theorem 4.1 For the appropriate choice of $\left\{T_{i}\right\}_{i=1}^{\infty}$, the knot $K_{T_{i}} \# K_{T_{j}}$ is not cg-slice, for $i \neq j$.

Proof The argument begins with some basic computations that follow readily from the techniques of [51] and [1]. Details are presented in [40]. The knot $K_{T}$ is built from $K_{0}$ by removing neighborhoods of curves $B_{1}$ and $B_{2}$ linking the bands and replacing them with the complements of $T$ and $-T$. The $2-$ fold branched cover of $K_{T}, M\left(K_{T}\right)$, has first homology $\mathbf{Z}_{5}$, and if we consider a 
map of $M\left(K_{T}\right)$ to $\mathbf{Z}_{5}$ taking value 1 on a lift of $B_{1}$, it takes value -1 on the other lift, and takes values 2 and -2 on the lifts of $B_{2}$.

The homology of the 2-fold cover of $K_{T_{i}} \# K_{T_{j}}$ is $\mathbf{Z}_{5} \oplus \mathbf{Z}_{5}$, and a careful examination of the linking form reveals that for any metabolizer, one of the characters that vanishes on that metabolizer will take value 1 on a generator of the first $\mathbf{Z}_{5}$ summand and \pm 2 on the corresponding generator of the other summand. (In brief, a metabolizing element $(a, b)$ must satisfy $a^{2}+b^{2}=0 \bmod 5$.)

Hence, a calculation similar to the one for doubled knots given in the previous section shows that for this character

$$
\sigma_{b}\left(K_{T_{i}} \# K_{T_{j}}\right)=\left(2^{k}\left(\sigma_{1 / 5}\left(T_{i}\right)-\sigma_{2 / 5}\left(T_{i}\right)+\sigma_{2 / 5}\left(T_{j}\right)-\sigma_{1 / 5}\left(T_{k}\right)\right)\right)_{k=1 \ldots \infty}
$$

An explicit calculation for the $(2,7)$-torus knot, $T_{1}$, gives that $\sigma_{1 / 5}\left(T_{1}\right)=-2$ and $\sigma_{2 / 5}\left(T_{1}\right)=-6$. Hence, if we let $T_{i}$ denote the connected sum of $i(2,7)-$ torus knots, one computes

$$
\sigma_{b}\left(K_{T_{i}} \# K_{T_{j}}\right)=\left(2^{k}(-2 i+6 i-6 j+2 j)\right)_{k=1 \ldots \infty}=\left(2^{k+2}(i-j)\right)_{k=1 \ldots \infty}
$$

Clearly, this last sequence is unbounded unless $i=j$.

Theorem 4.1 and Corollary 3.6 immediately imply the following.

Corollary 4.2 For the appropriate choice of knots $\left\{T_{i}\right\}_{i=1}^{\infty}$, the knots $K_{T_{i}} \# K_{0}$ generate an infinite 2-torsion subgroup of $\operatorname{ker} \phi_{2}: \mathcal{M} \rightarrow \mathcal{G}$.

\section{The kernel of $\phi_{1}$ contains $\mathrm{Z}^{\infty}$}

In Section 5.1 we will describe the construction of a knot, $K_{J}$, based on an arbitrary knot $J$. It follows from the construction that $K_{J}$ is slice; our focus will be on the positive mutants, $K_{J}^{*}$. For instance, Theorem 5.2 states that for some $J, K_{J}^{*}$ is not slice; this proves that the kernel of $\phi_{1}$ is nontrivial.

In order to apply Theorem 2.3 to $K_{J}^{*}$, and linear combinations of $K_{J}$, we need to understand the homology of the appropriate branched covers. The 3-fold cover is sufficient for our needs, and in Section 5.2 we discuss the homology of this space. This analysis can be done by considering the cover directly, but we will instead exploit a relationship between the homology of the 3-fold branched cover and metacyclic representations of the knot group. The advantage of this approach is that it simultaneously gives us information about the iterated covers used in defining the Casson-Gordon invariants. 
Section 5.3 presents the proof that for appropriate $J, K_{J}^{*}$ is not slice. In this section we also develop many of the basic tools and computations needed for the linear independence results of the following sections.

The last three sections, Sections 5.4, 5.5, and 5.6, are devoted to proving that the kernel contains $\mathbf{Z}^{\infty}$, generated by knots $K_{J}^{*}$ for appropriate $J$. Most of the work is focused on understanding the invariant metabolizers in the homology of the 3-fold branched covering spaces. If we consider the connected sum of $n$ of the $K_{J}^{*}$, the homology of the 3 -fold branched cover, working with $\mathbf{Z}_{7}$ coefficients, is $\left(\mathbf{Z}_{7} \oplus \mathbf{Z}_{7}\right)^{n}$. Under the $\mathbf{Z}_{3}$ action, this will be seen to split as a 2-eigenspace and a 4-eigenspace, each isomorphic to $\left(\mathbf{Z}_{7}\right)^{n}$. Metabolizers will similarly split, say for now as $M_{2}$ and $M_{4}$. The calculations of Section 5.3 will quickly imply that if either $M_{2}$ and $M_{4}$ contains an odd vector, meaning one with an odd number of nonzero coordinates, then the corresponding knot will not be slice. Simple linear algebra will show that if the metabolizer is not "evenly split" between $M_{2}$ and $M_{4}$ then this will be the case. The final case, in which the metabolizer contains no odd vectors is the most delicate. It calls on a careful examination of the linking form of the 3-fold branched cover, carried out in Section 5.5, along with some difficult linear algebra, carried out in Section 5.6.

\subsection{Building the examples, $K_{J}^{*}$}

Figure 4 illustrates the needed components of the construction of our examples. The figure includes a knot in $S^{3}, K$, and three unknotted circles, $B_{1}, B_{2}$ and $B_{2}^{*}$, in the complement of $K$. Also drawn are dotted lines, $\alpha_{1}$ and $\alpha_{2}$. Not drawn is a second knot, $J$, to be used throughout the construction. The final knots we construct will depend on the choice of $J$ and will be denoted $K_{J}$.

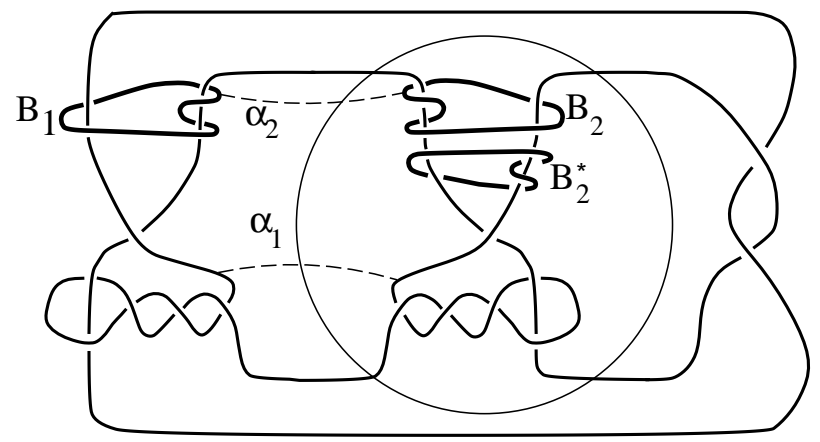

Figure 4: The Knot $K$ 
First we note that $K$ is a slice knot. This is seen by performing the band move to $K$ along the arc $\alpha_{1}$. A pair of unknotted and unlinked circles results, and so $K$ is slice.

The knot $K_{J}$ is formed by removing neighborhoods of $B_{1}$ and $B_{2}$ and replacing them with the complement of $J$ and $-J$, respectively. As usual, the meridian and longitude are interchanged. As explained in Section 2, the knot $K_{J}$ is an (iterated) satellite knot in $S^{3}$ since $B_{1}$ and $B_{2}$ are unknotted. We have:

Theorem $5.1 K_{J}$ is slice.

Proof First we note that $B_{1}$ and $B_{2}$ cobound an annulus $A$ embedded in the complement in $B^{4}$ of the slice disk for $K$ just constructed. To see this, perform the band move joining $B_{1}$ and $B_{2}$ along the arc $\alpha_{2}$. The resulting circle is unknotted and unlinked from the pair of circles formed during the slicing of $K$, so that circle can be capped off with a disk in the complement of the slice disk to yield $A$. We also need to note that $A$ is trivial when viewed as an annulus in $B^{4}$. That is, the pair $\left(B^{4}, A\right)$ is equivalent to the pair $\left(B^{3}, U\right) \times[0,1]$, where $U$ is the unknot. This is easily seen by ignoring $K$ and the slice disk during the construction of $A$.

It is clear that if one removes from $\left(B^{3}, U\right) \times[0,1]$ a neighborhood of $U \times[0,1]$ and replaces it with $\left(S^{3}-J\right) \times[0,1]$ then $B^{3} \times[0,1]=B^{4}$ results. The boundary, $S^{3}=\partial B^{4}$, is obtained from the original $S^{3}$ by removing neighborhoods of $B_{1}$ and $B_{2}$ and replacing them with the complement of $J$ and $-J$ respectively, as desired. During this construction, the slice disk for $K$ becomes the slice disk for $K_{J}$.

\section{The positive mutant of $K_{J}$}

A positive mutant of $K$ (or $K_{J}$ ) is formed by cutting along the sphere $S$, illustrated by the circle in Figure 4, and rotating 180 degrees about the vertical axis. It is not hard to show that the resulting mutant, $K^{*}$, of $K$ is isotopic to $K$. However, the mutant $K_{J}^{*}$ of $K_{J}$ is (as we will show) not concordant to $K_{J}$ for many choices of $J$. For now it should be apparent that $K_{J}^{*}$ is formed from $K$ by removing the unknots $B_{1}$ and $B_{2}^{*}$ and replacing them with the complements of $J$ and $-J$ respectively. We will ultimately prove:

Theorem 5.2 For appropriate choices of $J, K_{J}^{*}$ is not slice, and in particular $K_{J}^{*}$ is not concordant to $K_{J}$. 
By considering various choices for $J$ we will construct the infinite family of linear independent examples desired. The main result of this section is the following.

Theorem 5.3 There exists an infinite collection of knots $J_{1}, J_{2}, \ldots$ so that for any choice of integers $n_{1}, n_{2}, \ldots$ with only finitely many of the $n_{i}$ nonzero, the connected sum

$$
\#_{i} n_{i} K_{J_{i}}
$$

is slice, but

$$
\#_{i} n_{i} K_{J_{i}}^{*}
$$

is not slice. In particular the kernel of $\phi_{1}: \mathcal{C} \rightarrow \mathcal{M}$ contains a subgroup isomorphic to $\mathbf{Z}^{\infty}$.

\subsection{The homology of the 3 -fold cyclic cover of $K_{J}^{*}$}

The details concerning the homology of the 3 -fold cyclic branched cover of $K$ that we need are captured by the following theorem. Isolating out this detailed information permits us to avoid a complete analysis of the homology and cohomology, with group action, of the 3-fold cover.

\section{Theorem 5.4}

(a) The 3-fold cover of $S^{3}$ branched over $K, M_{3}$, satisfies $H_{1}\left(M_{3} ; \mathbf{Z}\right)=$ $\mathbf{Z}_{49} \oplus \mathbf{Z}_{49}$.

(b) The group $H^{1}\left(M_{3} ; \mathbf{Z}_{49}\right) \cong \mathbf{Z}_{49} \oplus \mathbf{Z}_{49}$ splits as the direct sum of two 1-dimensional eigenspaces, $C_{18}$ and $C_{30}$, under the action of the deck transformation $T$. (The eigenvalues are 18 and 30, the two nontrivial cube roots of unity in $\mathbf{Z}_{49}$.)

(c) There exists a choice of lifts, $\tilde{B}_{1}, \tilde{B}_{2}, \tilde{B}_{2}^{*}$, of the curves $B_{1}, B_{2}$, and $B_{2}^{*}$, and characters $\chi_{18} \in C_{18}$ and $\chi_{30} \in C_{30}$ (which generate these eigenspaces), so that $\chi_{i}\left(\tilde{B}_{1}\right)=1, \chi_{i}\left(\tilde{B}_{2}\right)=1$, and $\chi_{i}\left(\tilde{B}_{2}^{*}\right)=-1$ for $i=18,30$.

Proof The Alexander polynomial of $K$ is $\left(2 t^{2}-5 t+2\right)^{2}$. This can be quickly seen by noticing that changing one of the two rightmost crossings on $K$ in Figure 4 changes $K$ into the connected sum of two copies of the 3 -twisted double of the unknot, which has Alexander polynomial $2 t^{2}-5 t+2$. The Conway skein relation shows that changing this crossing does not change the Alexander polynomial, 
since the link obtained by smoothing the crossing is a unlink. It follows that the order of $H_{1}\left(M_{3} ; \mathbf{Z}\right)$ equals $\left(2 \zeta^{2}-5 \zeta+2\right)^{2}\left(2\left(\zeta^{2}\right)^{2}-5\left(\zeta^{2}\right)+2\right)^{2}=7^{4}$ (see, for example, [4, Theorem 8.21]). The assertion that $H_{1}\left(M_{3} ; \mathbf{Z}\right)=\mathbf{Z}_{49} \oplus \mathbf{Z}_{49}$ will follow once we show that $H^{1}\left(M_{3} ; \mathbf{Z}_{49}\right)=\mathbf{Z}_{49} \oplus \mathbf{Z}_{49}$.

The cube roots of 1 in $\mathbf{Z}_{49}$ are 1,18 and 30. The deck transformation $T$ acting on $H^{1}\left(M_{3} ; \mathbf{Z}_{49}\right)$ satisfies $T^{3}-1=0$. Hence, $(T-1)(T-18)(T-30)=0$. If $H^{1}\left(M_{3} ; \mathbf{Z}_{49}\right)^{\mathbf{Z}_{3}}$ denotes the fixed cohomology, then the composite

$$
H^{1}\left(M_{3} ; \mathbf{Z}_{49}\right)^{\mathbf{Z}_{3}} \stackrel{\tau^{*}}{\longrightarrow} H^{1}\left(S^{3} ; \mathbf{Z}_{49}\right) \stackrel{\pi^{*}}{\longrightarrow} H^{1}\left(M_{3} ; \mathbf{Z}_{49}\right)^{\mathbf{Z}_{3}}
$$

is just multiplication by 3 , where $\tau^{*}$ denotes the transfer and $\pi: M_{3} \rightarrow S^{3}$ is the branched cover (see eg [3, Section III.2]); hence $H^{1}\left(M_{3} ; \mathbf{Z}_{49}\right)^{\mathbf{Z}_{3}}=0$. Thus $T-1$ is injective on the finite group $H^{1}\left(M_{3} ; \mathbf{Z}_{49}\right)$ and hence an isomorphism. It follows that $(T-18)(T-30)=0$.

Let

$$
C_{18}=\operatorname{ker}(T-18): H^{1}\left(M_{3} ; \mathbf{Z}_{49}\right) \rightarrow H^{1}\left(M_{3} ; \mathbf{Z}_{49}\right)
$$

and

$$
C_{30}=\operatorname{ker}(T-30): H^{1}\left(M_{3} ; \mathbf{Z}_{49}\right) \rightarrow H^{1}\left(M_{3} ; \mathbf{Z}_{49}\right) .
$$

For any $x \in H^{1}\left(M_{3} ; \mathbf{Z}_{49}\right), x=(T-30)(4 x)+(T-18)(-4 x)$ so $H^{1}\left(M_{3} ; \mathbf{Z}_{49}\right)=$ $C_{18}+C_{30}$. If $x \in C_{18} \cap C_{30}$ then $30 x=18 x$, so $22 x=0$ which implies $x=0$ since 22 is relatively prime to 49 .

Hence, the cohomology does split as the direct sum of an 18- and a 30eigenspace, $H^{1}\left(M_{3} ; \mathbf{Z}_{49}\right)=C_{18} \oplus C_{30}$. We will show that these are each 1dimensional (ie, cyclic of order 49), by identifying elements in each eigenspace with certain metabelian representations of $\pi_{1}\left(S^{3}-K\right)$.

Define the metacyclic group

$$
G=\left\langle t, r \mid t^{3}=1, r^{49}=1, t r=r^{30} t\right\rangle .
$$

Consider the elements of $H^{1}\left(M_{3} ; \mathbf{Z}_{49}\right)$ as homomorphisms $\chi: \pi_{1}\left(M_{3}\right) \rightarrow \mathbf{Z}_{49}$. For each $\chi \in C_{30}$, we construct a representation $\bar{\chi}: \pi_{1}\left(S^{3}-K\right) \rightarrow G$ taking value $r^{i} t$ on meridians, as follows.

Pick a basepoint for $\pi_{1}\left(S^{3}-K\right)$ on the boundary of a meridinal disk for $K$ and choose the basepoint of $M_{3}$ to lie above it, and hence on the boundary of a meridinal disk for the branch set in $M_{3}$. We can then lift a loop $\alpha \in \pi_{1}\left(S^{3}-K\right)$ to a loop $\tilde{\alpha}$ in $\pi_{1}\left(M_{3}\right)$ by letting $\tilde{\alpha}$ be the lift of $\alpha$ followed (if necessary) by an arc back to the basepoint in the meridinal disk in $M_{3}$. Let $|\cdot|: \pi_{1}\left(S^{3}-K\right) \rightarrow \mathbf{Z}_{3}$ be the homomorphism taking the meridian of $K$ to $1 \in \mathbf{Z}_{3}$. 
To a class $\chi \in C_{30}$ we assign a representation $\bar{\chi}$ on $\pi_{1}\left(S^{3}-K\right)$ by the formula

$$
\bar{\chi}(\alpha)=r^{\chi(\tilde{\alpha})} t^{|\alpha|} .
$$

If $\mu \in \pi_{1}\left(S^{3}-K\right)$ denotes the meridian of $K$ then $\bar{\chi}\left(\mu^{3}\right)=1$. The projection induces an inclusion $\pi_{1}\left(M_{3}\right) \rightarrow \pi_{1}\left(S^{3}-K\right) /\left\langle\mu^{3}=1\right\rangle$ and so we can recover $\chi$ by restricting $\bar{\chi}$ to $\pi_{1}\left(M_{3}\right)$; its image lies in the $\mathbf{Z}_{49}$ subgroup of $G$ generated by $r$.

To turn this into a one-to-one correspondence, one needs to identify representations of $\pi_{1}\left(S^{3}-K\right)$ to $G$ that are equivalent on the $\mathbf{Z}_{49}$ subgroup. These are precisely the conjugacy classes of representations.

Figure 5 below describes a representation of $\pi_{1}\left(S^{3}-K\right)$ to $G$. The edges of the knot diagram (ie, the Wirtinger generators of $\pi_{1}\left(S^{3}-K\right)$ ) are labeled with numbers in $\mathbf{Z}_{49}$, where the label " $b$ " means the corresponding Wirtinger generator is sent to the element $r^{b} t$ in $G$. To give a well defined representation, the Wirtinger relation $x_{i} x_{j} x_{i}^{-1}=x_{k}$ at a crossing implies that the relation $r^{b_{i}} r^{b_{j}} t\left(r^{b_{i}} t\right)^{-1}=r^{b_{k}} t$ must hold, where $b_{\ell}$ denotes the label on the $\ell$ th Wirtinger generator. This relation is satisfied if and only if the labels satisfy $29 b_{i}-30 b_{j}+b_{k}=0$ at each crossing.

Observe that conjugation by $r$ takes $r^{i} t$ to $r^{i-1} t$ and so in enumerating conjugacy classes of representations (and hence $C_{30}$ ), we can assume that any one label of the diagram is 0 . Or, put otherwise, two labelings determine conjugate representations if all corresponding labels differ by the same amount. It is a simple exercise to check that all labelings are obtained from the labeling of Figure 5 by either multiplying each label by $a \in \mathbf{Z}_{49}$ or by adding the same number to each.

It follows that $C_{30}$ is isomorphic to $\mathbf{Z}_{49}$, generated by the character $\chi$ determined by the labeling in Figure 5. A similar computation applies for $C_{18}$. Hence $H^{1}\left(M_{3} ; \mathbf{Z}_{49}\right)=\mathbf{Z}_{49} \oplus \mathbf{Z}_{49}$.

To prove part (c) one needs only compute the value of the representation $\bar{\chi}$ on the $B_{i}$. In Figure 5 we have chosen based loops to represent the curves $B_{1}, B_{2}$, and $B_{2}^{*}$. The basing determines specific lifts $\tilde{B}_{1}, \tilde{B}_{2}$, and $\tilde{B}_{2}^{*}$ of these curves to $M_{3}$.

For the representation given by the labeling in Figure 5 it is easy to compute that $\bar{\chi}\left(B_{1}\right)=r, \bar{\chi}\left(B_{2}\right)=r$, and $\bar{\chi}\left(B_{2}^{*}\right)=r^{-1}$. Hence the corresponding character $\chi_{30} \in C_{30} \subset H^{1}\left(M_{3} ; \mathbf{Z}_{49}\right)$ satisfies $\chi_{30}\left(\tilde{B}_{1}\right)=1, \chi_{30}\left(\tilde{B}_{2}\right)=1$, and $\chi_{30}\left(\tilde{B}_{2}^{*}\right)=-1$. A similar argument applies to $\chi_{18} \in C_{18}$. 


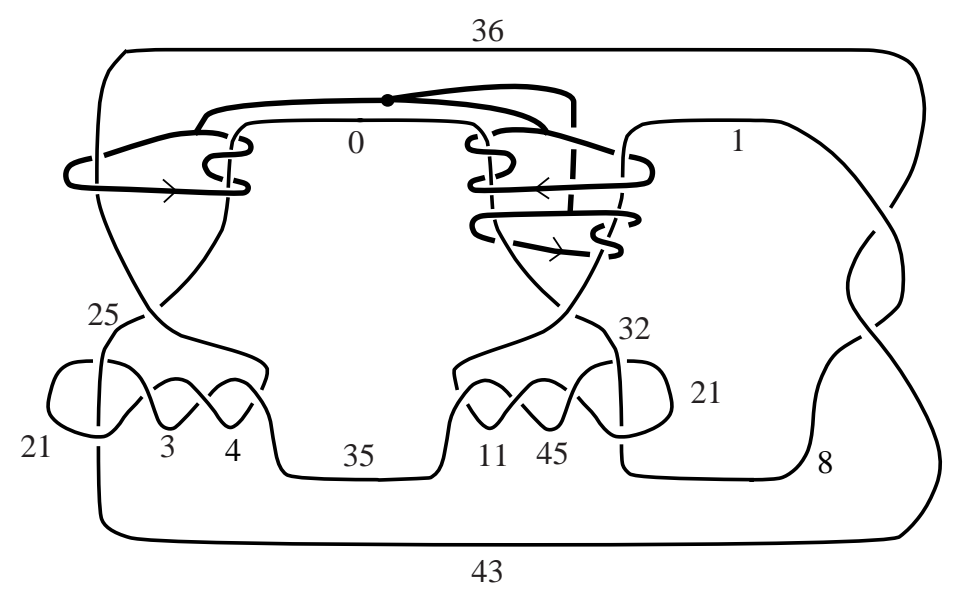

Figure 5: The representation $\bar{\chi}: \pi_{1}\left(S^{3}-K\right) \rightarrow G$

Given a knot $J$ in $S^{3}$, we will use the notation $M_{3}(J)$ to denote the 3 -fold branched cover of $J$.

Corollary 5.5 The 3-fold branched cover $M_{3}\left(K_{J}^{*}\right)$ of $K_{J}^{*}$ has the same homology and cohomology as as the 3-fold branched cover $M_{3}=M_{3}(K)$ of $K$; there is a natural correspondence between characters on $M_{3}(K)$ and characters on $M_{3}\left(K_{J}^{*}\right)$. In particular, the characters $\chi_{i}$ of Theorem 5.4 extend to $M_{3}\left(K_{J}^{*}\right)$ and $H^{1}\left(M_{3}\left(K_{J}^{*}\right) ; \mathbf{Z}_{49}\right)=C_{18} \oplus C_{30}$.

Proof The curves $B_{1}, B_{2}^{*}$ each lift to three curves in $M_{3}$, and the MayerVietoris sequence shows that replacing the six solid tori lying above these curves with knot complements, $S^{3}-J$ or $S^{3}-(-J)$, does not affect the cohomology.

For most of our work we will only need to work modulo 7 instead of modulo 49. Thus we consider the inclusion $\mathbf{Z}_{7} \rightarrow \mathbf{Z}_{49}$ induced by $1 \mapsto 7$. This induces an injection $H^{1}\left(M_{3} ; \mathbf{Z}_{7}\right) \rightarrow H^{1}\left(M_{3} ; \mathbf{Z}_{49}\right)$ with image $7 H^{1}\left(M_{3} ; \mathbf{Z}_{49}\right)$. We will always identify $H^{1}\left(M_{3} ; \mathbf{Z}_{7}\right)$ with $7 H^{1}\left(M_{3} ; \mathbf{Z}_{49}\right)$ in this way. Let $C_{2}=C_{30} \cap$ $H^{1}\left(M_{3} ; \mathbf{Z}_{7}\right)$ and let $C_{4}=C_{18} \cap H^{1}\left(M_{3} ; \mathbf{Z}_{7}\right)$. Then $C_{2}$ and $C_{4}$ are 2 and 4 eigenspaces of the action of $\mathbf{Z}_{3}$ on $H^{1}\left(M_{3} ; \mathbf{Z}_{7}\right)$; each is 1-dimensional, and $H^{1}\left(M_{3} ; \mathbf{Z}_{7}\right)=C_{2} \oplus C_{4}$. We let $\chi_{2}=7 \chi_{30}$ and $\chi_{4}=7 \chi_{18}$; thus $\chi_{2}$ generates $C_{2}$ and $\chi_{4}$ generates $C_{4}$ and $\chi_{i}\left(B_{1}\right)=1=\chi_{i}\left(B_{2}\right)$ and $\chi_{i}\left(B_{2}^{*}\right)=-1$ (in $\mathbf{Z}_{7}$ ) for $i=2,4$. Until Section 5.5 we will work $\bmod 7$. 


\section{$5.3 \quad K_{J}^{*}$ is not slice}

The proof that the kernel of $\phi_{1}$ is infinitely generated will depend on constructing an infinite family of knots from $K$ by using different choices of $J$. To introduce some of the key ingredients we will first prove Theorem 5.2, stating that for an appropriate choice of $J, K_{J}^{*}$ is not slice.

Proof of Theorem 5.2 We will determine the needed properties of $J$ in the course of the argument.

If $K_{J}^{*}$ is slice, by Theorem 2.4 there is some metabolizer $A \subset H_{1}\left(M_{3}\left(K_{J}^{*}\right)\right)$ which is invariant under $T$ and for which the appropriate Casson-Gordon invariants (certain $\delta$ ) will vanish. Simply by order considerations there will be a nontrivial $\mathbf{Z}_{7}$ valued character, $\chi \in H^{1}\left(M_{3}\left(K_{J}^{*}\right) ; \mathbf{Z}_{7}\right)$, that vanishes on $A$. (The quotient, $H_{1}\left(M_{3}\left(K_{J}^{*}\right)\right) / A$ contains 7 -torsion and hence admits a surjection to $\mathbf{Z}_{7}$.) The set $A^{*}$ of all elements in $H^{1}\left(M_{3}\left(K_{J}^{*}\right) ; \mathbf{Z}_{7}\right)$ that vanish on $A$ is $T$-invariant since $A$ is, so $A^{*}$ either is 1 -dimensional and generated by an eigen-character, or $A^{*}$ is all of $H^{1}\left(M_{3}\left(K_{J}^{*}\right) ; \mathbf{Z}_{7}\right)$. In either case, one of $\chi_{2}$ or $\chi_{4}$ must be in $A^{*}$. We denote it simply by $\chi$ below.

The Casson-Gordon invariant $\delta\left(K_{J}^{*}, \chi\right)$ associated to $\chi$ was defined in Section 2.3. Notice that $M_{3}\left(K_{J}^{*}\right)$ decomposes into 7 pieces, the 3 -fold cover branched cover of $S^{3}-\left(B_{1} \cup B_{2}^{*}\right)$ branched over $K, 3$ path components homeomorphic to $S^{3}-J$ lying over $B_{1}$, and 3 path components homeomorphic to $S^{3}-(-J)$ lying over $B_{2}^{*}$.

We claim that

$$
\delta\left(K_{J}^{*}, \chi\right)=\delta(K, \chi) \Delta_{J}(\zeta t) \Delta_{J}\left(\zeta^{2} t\right) \Delta_{J}\left(\zeta^{4} t\right) \Delta_{J}\left(\zeta^{-1} t\right) \Delta_{J}\left(\zeta^{-2} t\right) \Delta_{J}\left(\zeta^{-4} t\right) .
$$

where

(1) $\Delta_{J}$ is the Alexander polynomial of $J$.

(2) $\zeta$ is a primitive 7 -root of unity.

This formula follows from several applications of Theorem 2.9 and the following observation. If $\chi$ takes value 1 on some lift of $B_{1}$, it takes values 2 and 4 on the other lifts (if $\chi \in C_{2}$ then $\chi\left(T \tilde{B}_{1}\right)=2 \chi\left(\tilde{B}_{1}\right)=2$ and $\chi\left(T^{2} \tilde{B}_{1}\right)=4 \chi\left(\tilde{B}_{1}\right)=4$; similarly for $\chi \in C_{4}$, since $\left.4^{2}=2(\bmod 7)\right)$. This explains the appearance of the terms $\Delta_{J}(\zeta t), \Delta_{J}\left(\zeta^{2} t\right), \Delta_{j}\left(\zeta^{4} t\right)$. We argue similarly for $B_{2}^{*}$ using the fact that the Alexander polynomial of $-J$ is the same as the polynomial for $J$ and that $\chi$ takes the value -1 on $\tilde{B}_{2}^{*}$.

There is a tricky point here. If the orientation of $B_{1}$ is changed then $\chi$ will take value -1 on the lift of $B_{1}$. However, the representation of the homology 
of the cyclic cover of $S^{3}-K$ to $\mathbf{Z}$ used to define $\delta$ will take value $-1 \in \mathbf{Z}$ rather than 1 . Hence the term $\Delta_{J}(\zeta t)$ will be replaced with $\Delta_{J}\left(\zeta^{-1} t^{-1}\right)$. But this just equals $\Delta_{J}\left((\zeta t)^{-1}\right)$, and by the symmetry of the Alexander polynomial the term is unchanged.

Our first conditions on $\Delta_{J}$ (and hence on $J$ ) is that it be irreducible in $\mathbf{Q}(\zeta)\left[t, t^{-1}\right]$. This is easily attained: let $J$ be a knot having Alexander polynomial $a t^{2}+(1-2 a) t+a$. This is irreducible since the roots are complex, and as long as its discriminant $(1-4 a)$ is not divisible by 7 it will not factor in $\mathbf{Q}(\zeta)\left[t, t^{-1}\right]$ (this is shown in greater generality in the next section).

A second condition on $\Delta_{J}$ is that it be relatively prime to $\delta(K, \chi)$ in $\mathbf{Q}(\zeta)\left[t, t^{-1}\right]$ for each $\chi \in H^{1}\left(M_{3} ; \mathbf{Z}_{7}\right)$. But since $\delta(K, \chi)$ has only a finite number of irreducible factors, and $H^{1}\left(M_{3} ; \mathbf{Z}_{7}\right)$ has 49 elements, this too is easy to achieve.

With these conditions, it is clear that $\delta\left(K_{J}^{*}, \chi\right)$ can be a norm (that is, of the form $g(t) \overline{g(t)}$ ) only if

$$
S(t)=\Delta_{J}(\zeta t) \Delta_{J}\left(\zeta^{2} t\right) \Delta_{J}\left(\zeta^{4} t\right) \Delta_{J}\left(\zeta^{-1} t\right) \Delta_{J}\left(\zeta^{-2} t\right) \Delta_{J}\left(\zeta^{-4} t\right)
$$

is a norm.

We next note that the six factors in this product are irreducible and are pairwise relatively prime in $\mathbf{Q}(\zeta)\left[t, t^{-1}\right]$. Certainly the change of variable cannot make them reducible. If two were not relatively prime they would be associates; one would be an nontrivial $\mathbf{Q}(\zeta)$ multiple of the other. But this is immediately checked to not be the case.

So, suppose that $S(t)$ is of the form $g(t) \overline{g(t)}$. Notice that $\overline{\Delta\left(\zeta^{i} t\right)}=\Delta\left(\zeta^{-i} t^{-1}\right)=$ $\Delta\left(\left(\zeta^{i} t\right)^{-1}\right)$. However, by the symmetry of the Alexander polynomial, this last term is simply $\Delta\left(\zeta^{i} t\right)$ (modulo a multiple of the form $t^{2}$ which is itself a norm). It follows that for $S(t)$ to be a norm, it would have to be a square; since each irreducible factor is distinct, this is not the case. Thus $K_{J}^{*}$ is not slice.

We formalize the last paragraph of this proof in the following useful algebraic lemma.

Lemma 5.6 Let $q_{1}(t), q_{2}(t), \ldots, q_{n}(t)$ be integer Laurent polynomials. Let $\zeta=e^{2 \pi i / 7}$. Suppose that $q_{i}(t)=q_{i}\left(t^{-1}\right)$, that each $q_{i}(t)$ is irreducible in $\mathbf{Q}(\zeta)\left[t, t^{-1}\right]$, and that the $q_{i}(t)$ are relatively prime in $\mathbf{Q}\left[t, t^{-1}\right]$. Also assume that none of the $q_{i}$ can be written as $t^{m} f\left(t^{7}\right)$ for some $f(t) \in \mathbf{Q}(\zeta)\left[t, t^{-1}\right]$ and integer $m$. 
Suppose that $k_{1}, k_{2}, \ldots, k_{n}$ are non-negative integers and that $a_{i j} \in \mathbf{Z}_{7}, i=$ $1,2, \ldots, n, j=1,2, \ldots, k_{i}$, and let

$$
R(t)=\left(\prod_{j=1}^{k_{1}} q_{1}\left(\zeta^{a_{1 j}} t\right)\right) \cdots\left(\prod_{j=1}^{k_{n}} q_{n}\left(\zeta^{a_{n j}} t\right)\right) .
$$

Then $R(t)$ is a norm in $\mathbf{Q}(\zeta)\left[t, t^{-1}\right]$ if and only if each $a \in \mathbf{Z}_{7}$ appears an even number of times in the vector $\left(a_{\ell 1}, a_{\ell 2}, \ldots, a_{\ell k_{\ell}}\right)$ for each $\ell \in\{1,2, \ldots, n\}$.

Proof Each irreducible factor of $R(t)$ is of the form $q_{i}\left(\zeta^{j} t\right)$ for some $i$ and $j$,

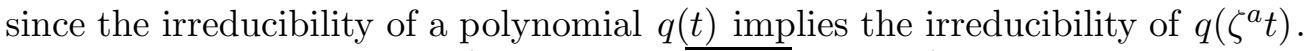
Each of these factors, $q_{i}\left(\zeta^{j} t\right)$, satisfies $\overline{q_{i}\left(\zeta^{j} t\right)}=q_{i}\left(\zeta^{j} t\right)$. Hence, if $R(t)=$ $Q(t) \overline{Q(t)}$ for some $Q(t)$, then the exponent of each irreducible factor of $R(t)$ must be even.

It remains to observe that the set of polynomials $\left\{q_{i}\left(\zeta^{j} t\right)\right\}_{i=1, \ldots, n, j=0, \ldots, 6}$ are distinct. Suppose that $q_{\alpha}\left(\zeta^{r} t\right)=q_{\beta}\left(\zeta^{s} t\right)$. Then a change of variable shows that $q_{\alpha}(t)=q_{\beta}\left(\zeta^{s-r} t\right)$. Since the $q$ are integer polynomials, the only way these can be equal is if $s-r=0$. (This is where we use the fact that the $q_{i}(t)$ are not polynomials in $t^{7}$.) But this results in the equation $q_{\alpha}(t)=q_{\beta}(t)$, implying that $\alpha=\beta$.

\subsection{Case I: Metabolizers with odd characters}

We turn now to the proof of Theorem 5.3, that is, to showing that the kernel of $\phi_{1}$ contains $\mathbf{Z}^{\infty}$. There are basically two separate cases in the argument, depending on whether or not the space of characters that vanish on a given metabolizer contains an odd vector, to be defined below.

Let $\mathcal{S}=\left\{S_{1}, S_{2}, \cdots\right\}$ be an infinite collection of isotopy classes of oriented knots in $S^{3}$ so that their Alexander polynomials $\Delta_{S_{i}}(t)$ satisfy

(1) $\Delta_{S_{i}}(t)$ is irreducible in $\mathbf{Q}(\zeta)\left[t, t^{-1}\right]$.

(2) $\Delta_{S_{i}}(t)$ is relatively prime to $\Delta_{S_{j}}(t)$ for all $i, j$.

(3) $\Delta_{S_{i}}(t)$ is not of the form $t^{m} f\left(t^{7}\right)$ for some $m$ and rational polynomial $f$.

(4) $\Delta_{S_{i}}\left(\zeta^{a} t\right)$ is relatively prime to the 49 elements $\delta(K ; \chi), \chi \in H^{1}\left(M_{3} ; \mathbf{Z}_{7}\right)$.

Such a collection is easily constructed. Consider for instance the polynomial $S(t)=a t^{2}-(2 a+1) t-a, a>0$. If $a$ is not of the form $r(r+1)$ for some integer $r$, then $S(t)$ is irreducible and has real roots $\frac{(2 a+1) \pm \sqrt{4 a+1}}{2 a}$. If $S(t)$ were reducible 
over $\mathbf{Q}\left(\zeta_{7}\right)$, then we would have the extension $\mathbf{Q} \subset \mathbf{Q}(\sqrt{4 a+1}) \subset \mathbf{Q}\left(\zeta_{7}\right)$. However, $\mathbf{Q}\left(\zeta_{7}\right)$ is a degree 6 Galois extension of $\mathbf{Q}$ and hence contains a unique degree 2 extension of $\mathbf{Q}$. As we show next, that extension is not real while $\mathbf{Q}(\sqrt{4 a+1})$ is, leading to the desired contradiction.

To see that the degree 2 extension $F$ of $\mathbf{Q}$ contained in $\mathbf{Q}\left(\zeta_{7}\right)$ is complex, proceed as follows. We have that $F$ is the fixed set of the order 3 Galois automorphism $\sigma$ of $\mathbf{Q}\left(\zeta_{7}\right)$, defined by $\sigma\left(\zeta_{7}\right)=\zeta_{7}^{2}$. A fixed element of $\sigma$ is $\alpha=\zeta_{7}+\zeta_{7}^{2}+\zeta_{7}^{4}$. One checks that $\alpha$ satisfies $t^{2}+t+2=0$, so $\alpha=\frac{-1+\sqrt{-7}}{2}$ and $F=\mathbf{Q}(\sqrt{-7})$.

So, to construct the $S_{i}$ pick an infinite sequence of integers $a$ as above. The resulting polynomials will satisfy the first three conditions and all but a finite number of them satisfy the last condition. These polynomials occur as the Alexander polynomials of twisted doubles of the unknot.

Next suppose we are given

(1) a positive number $n$,

(2) a choice of knots $J_{i}, i=1, \ldots, n$ such that each $J_{i} \in \mathcal{S}$, and

(3) a choice of signs $\epsilon_{i} \in\{ \pm 1\}$ for $i=1, \ldots, n$, such that if $J_{i}=J_{j}$ then $\epsilon_{i}=\epsilon_{j}$.

Denote by $L$ the connected sum of the $n$ (oriented) knots

$$
L=\epsilon_{1} K_{J_{1}}^{*} \# \epsilon_{2} K_{J_{2}}^{*} \# \cdots \# \epsilon_{n} K_{J_{n}}^{*}
$$

and let $M_{3}(L)$ denote 3 -fold branched cover of $L$. Notice that performing $n$ mutations transforms $L$ into the slice knot $\epsilon_{1} K_{J_{1}} \# \epsilon_{2} K_{J_{2}} \# \cdots \# \epsilon_{n} K_{J_{n}}$, and hence $L$ lies in the kernel of $\phi_{1}: \mathcal{C} \rightarrow \mathcal{M}$.

We will show that $L$ is not slice by analyzing characters $\chi$ in $H^{1}\left(M_{3}(L) ; \mathbf{Z}_{7}\right)$. This will show that the kernel of $\phi_{1}$ contains an infinite direct sum of $\mathbf{Z}$.

The 3-fold branched cover of $S^{3}$ branched over $L, M_{3}(L)$, is the connected sum of $n$ copies of $M_{3}\left(\epsilon_{i} K_{J_{i}}^{*}\right)$, since the branched cover of $S^{2}$ branched over 2 points is a $2-$ sphere. Thus

$$
H^{1}\left(M_{3}(L) ; \mathbf{Z}_{7}\right)=\oplus_{i=1}^{n} H^{1}\left(M_{3}\left(\epsilon_{i} K_{J_{i}}^{*}\right) ; \mathbf{Z}_{7}\right) .
$$

At this point we have identified $H_{1}\left(M_{3}(K)\right)$ with $H_{1}\left(M_{3}\left(K_{J}^{*}\right)\right)$. We also need to identify $H_{1}\left(M_{3}\left(K_{J}^{*}\right)\right)$ with $H_{1}\left(M_{3}\left(-K_{J}^{*}\right)\right)$, along with their $\mathbf{Z}_{3}$ structures. First, since $-\left(S^{3}, K\right)=\left(-S^{3},-K\right), M_{3}\left(K_{J}^{*}\right)=-M_{3}\left(-K_{J}^{*}\right)$. Thus their first homologies are naturally identified. Next, we need to check that the $\mathbf{Z}_{3}$ actions agree under this identification. This follows from the fact that an oriented curve 
in $S^{3}-K$ that links $K$ algebraically once also links $-K$ algebraically once in $-S^{3}$. In particular, $\delta\left(K_{J}^{*}, \chi\right)=\delta\left(-K_{J}^{*}, \chi\right)$ for any $J$.

The covering transformation acts diagonally in the sum of Equation (1), hence we can write

$$
H^{1}\left(M_{3}(L) ; \mathbf{Z}_{7}\right)=\oplus_{i=1}^{n}\left(C_{2} \oplus C_{4}\right),
$$

and characters $\chi$ in $H^{1}\left(M_{3}(L) ; \mathbf{Z}_{7}\right)$ will be expressed as an $n$-tuple

$$
\chi=\left(a_{1} \chi_{2}+b_{1} \chi_{4}, a_{2} \chi_{2}+b_{2} \chi_{4}, \cdots, a_{n} \chi_{2}+b_{n} \chi_{4}\right) .
$$

Lemma 5.7 For the character $\chi \in H^{1}\left(M_{3}(L) ; \mathbf{Z}_{7}\right)$ of Equation (2), the Casson-Gordon discriminant $\delta(L ; \chi)$ equals

$$
\begin{aligned}
& \prod_{i=1}^{n}\left(\delta_{i} \cdot \Delta_{J_{i}}\left(\zeta^{a_{i}+b_{i}} t\right) \Delta_{J_{i}}\left(\zeta^{2 a_{i}+4 b_{i}} t\right) \Delta_{J_{i}}\left(\zeta^{4 a_{i}+2 b_{i}} t\right)\right. \\
& \left.\Delta_{J_{i}}\left(\zeta^{-a_{i}-b_{i}} t\right) \Delta_{J_{i}}\left(\zeta^{-2 a_{i}-4 b_{i}} t\right) \Delta_{J_{i}}\left(\zeta^{-4 a_{i}-2 b_{i}} t\right)\right)
\end{aligned}
$$

where $\Delta_{J_{i}}(t)$ denotes the Alexander polynomial of the knot $J_{i}$ and $\delta_{i}=$ $\delta\left(K, a_{i} \chi_{2}+b_{i} \chi_{4}\right)$.

Proof Using Theorem 5.4 one sees that since the character $a_{i} \chi_{2}+b_{i} \chi_{4}$ takes the value $a_{i}+b_{i}$ on the lift $\tilde{B}_{1}$ of $B_{1}$, it takes the value $2 a_{i}+4 b_{i}$ on the translate $T \tilde{B}_{1}$ and takes the value $4 a_{i}+2 b_{i}$ on $T^{2} \tilde{B}_{1}$.

Similarly $a_{i} \chi_{2}+b_{i} \chi_{4}$ takes the value $-a_{i}-b_{i}$ on $\tilde{B}_{2}^{*}$ and hence takes the value $-2 a_{i}-4 b_{i}$ on $T \tilde{B}_{2}^{*}$ and $-4 a_{i}-2 b_{i}$ on $T^{2} \tilde{B}_{2}^{*}$.

The lemma now follows from Theorem 2.9.

To show that $L$ is not slice, we will prove that for each invariant metabolizer $A \subset H_{1}\left(M_{3}(L) ; \mathbf{Z}\right)$ there exists a $\mathbf{Z}_{7}$ character $\chi$ vanishing on $A$ for which $\delta(L, \chi) \in \mathbf{Q}(\zeta)\left[t, t^{-1}\right]$ is not a norm.

Given a metabolizer $A \subset H_{1}\left(M_{3}(L)\right)$ which is invariant under the $\mathbf{Z}_{3}$ action induced by the covering transformation, let $A^{*} \in H^{1}\left(M_{3}(L) ; \mathbf{Z}_{7}\right)$ denote the subgroup of $\mathbf{Z}_{7}$ characters vanishing on $A$ (we identify $H^{1}\left(M_{3}(L) ; \mathbf{Z}_{7}\right.$ ) with $\left.\operatorname{Hom}\left(H_{1}\left(M_{3}(L)\right) ; \mathbf{Z}_{7}\right)\right)$. Since $A$ is $\mathbf{Z}_{3}$ invariant, so is $A^{*}$, hence $A^{*}$ is spanned by eigenvectors of the $\mathbf{Z}_{3}$ action. Hence $A^{*}$ decomposes into 2 and 4 eigenspaces (as before, the 1 eigenspace is trivial since $H^{1}\left(S^{3} ; \mathbf{Z}_{7}\right)=0$ ):

$$
A^{*}=A_{2}^{*} \oplus A_{4}^{*} .
$$

For $i=2$ or 4 , any character $\chi$ in $A_{i}^{*}$ can be written in the form $\chi=$ $\left(a_{1} \chi_{i}, a_{2} \chi_{i}, \cdots, a_{n} \chi_{i}\right)$. 
Definition 5.8 An eigen-character $\chi=\left(a_{1} \chi_{i}, a_{2} \chi_{i}, \cdots, a_{n} \chi_{i}\right)$ in $A_{i}^{*}$ is called odd if an odd number of the coefficients $a_{j}$ are non-zero.

Lemma 5.9 If $\chi \in A_{i}^{*}$ is odd, then $\delta(L, \chi)$ is not a norm in $\mathbf{Q}(\zeta)\left[t, t^{-1}\right]$.

Proof Given $a \in \mathbf{Z}_{7}$, the set

$$
\left\{\zeta^{a}, \zeta^{2 a}, \zeta^{4 a}, \zeta^{-a}, \zeta^{-2 a}, \zeta^{-4 a}\right\}
$$

equals $\left\{\zeta, \zeta^{2}, \zeta^{3}, \zeta^{4}, \zeta^{5}, \zeta^{6}\right\}$ if $a \neq 0$, and equals $\{1,1,1,1,1,1\}$ if $a=0$.

Suppose $\chi=\left(a_{1} \chi_{2}, a_{2} \chi_{2}, \cdots, a_{n} \chi_{2}\right) \in A_{2}^{*}$. Then by Lemma 5.7 and the choice of $J$

$\delta(L, \chi)=\delta \prod_{i \mid a_{i}=0} \Delta_{J_{i}}(t)^{6} \prod_{i \mid a_{i} \neq 0} \Delta_{J_{i}}(\zeta t) \Delta_{J_{i}}\left(\zeta^{2} t\right) \Delta_{J_{i}}\left(\zeta^{3} t\right) \Delta_{J_{i}}\left(\zeta^{4} t\right) \Delta_{J_{i}}\left(\zeta^{5} t\right) \Delta_{J_{i}}\left(\zeta^{6} t\right)$

Where $\delta=\prod_{i} \delta\left(K, a_{i} \chi\right)$.

By Lemma 5.6 $\Delta_{J_{i}}(t)^{6}$ is a norm. Using the hypotheses on $\Delta_{J_{i}}$, Lemma 5.6, and the fact that $\chi$ is odd, we see that, modulo norms, $\delta(L, \chi)$ equals a product

$$
\delta \cdot \prod_{r \in B} \Delta_{S_{r}}(\zeta t) \Delta_{S_{r}}\left(\zeta^{2} t\right) \Delta_{S_{r}}\left(\zeta^{3} t\right) \Delta_{S_{r}}\left(\zeta^{4} t\right) \Delta_{S_{r}}\left(\zeta^{5} t\right) \Delta_{S_{r}}\left(\zeta^{6} t\right)
$$

where $B \subset\{1,2, \ldots\}$ is the set of those $r$ so that the set $\left\{i \mid J_{i}=S_{r}\right.$ and $\left.a_{i} \neq 0\right\}$ contains an odd number of elements.

Since $\chi$ is odd, $B$ is non-empty and so by Lemma 5.6 the expression in Equation (1) is not a norm.

The same argument applies to $A_{4}^{*}$.

Corollary 5.10 Given a metabolizer $A$, if one of $A_{2}$ or $A_{4}$ contains an odd character $\chi$ then $\delta(L, \chi)$ is not a norm .

In preparation for the next lemma, notice that $A^{*} \subset H^{1}\left(M_{3}(L) ; \mathbf{Z}_{7}\right)=\left(\mathbf{Z}_{7}\right)^{2 n}$ always has dimension at least $n$. This follows from the fact that the metabolizer $A \subset H_{1}\left(M_{3}(L)\right)=\left(\mathbf{Z}_{49}\right)^{2 n}$ has order $\sqrt{\mid\left(\mathbf{Z}_{49}\right)^{2 n \mid}}=49^{n}$, and so

$$
A^{*}=\operatorname{Hom}\left(H_{1}\left(M_{3}(L)\right) / A, \mathbf{Z}_{7}\right)=\operatorname{Hom}\left(\left(\mathbf{Z}_{49}\right)^{2 n} / A ; \mathbf{Z}_{7}\right)
$$

has dimension at least $n$.

Lemma 5.11 If one of $A_{2}^{*}$ or $A_{4}^{*}$ has dimension greater than $n / 2$, then it contains an odd character. 
Proof Suppose that $A_{\ell}^{*}$ has dimension $k>n / 2$. Choose some basis for $A_{\ell}^{*}$. Each basis element is written $a^{i}=\left(a_{1}^{i} \chi_{\ell}, a_{2}^{i} \chi_{\ell}, \cdots, a_{n}^{i} \chi_{\ell}\right)$ which we consider as the $i$ th row of a $k \times n$ matrix $M$.

Elementary row operations, and interchanging of columns, which does not affect whether a row is odd (ie, has an odd number of non-zero entries) transforms the matrix $M$ to a matrix of the form $(I B)$, where $I$ is a $k \times k$ identity matrix and $B$ is a $k \times(n-k)$ matrix. If some row of $B$ is not odd, then the corresponding row of $(I B)$ is odd and we have the desired odd character.

Thus assume every row of $B$ is odd. Since $B$ has more rows than columns, some non-trivial linear combination of the rows of $B$ gives the zero vector, say $\sum_{i} s_{i} \operatorname{row}_{i}(B)=0$. If an odd number of the $s_{i}$ are non-zero, then $\sum_{i} s_{i} \operatorname{row}_{i}(I B)$ is odd.

Finally, if an even number of the $s_{i}$ are nonzero, choose $j$ so that $s_{j} \neq 0$, and pick $f \in \mathbf{Z}_{7}$ non-zero and different from $s_{j}$. Then $-f \cdot \operatorname{row}_{j}(I B)+$ $\sum_{i} s_{i} \operatorname{row}_{i}(I B)$ is odd since $f \cdot \operatorname{row}_{j}(B)$ is odd.

Remark Notice that the proof also works if $A_{\ell}^{*}$ has dimension $n / 2$ and the square matrix $B$ is singular. We will use this extension below.

If $n$ is odd, then for each $A$ one of $A_{2}^{*}$ and $A_{4}^{*}$ has dimension greater than $n / 2$, and hence contains an odd character. This shows $n K_{J}^{*}$ is not slice for $n$ odd or, more generally, that $L$ is not slice if $n$ is odd. But when $n$ is even there are some remaining cases when neither $A_{i}^{*}$ contains an odd vector. For example, consider the situation when $L=K_{S_{1}}^{*} \# K_{S_{1}}^{*}$ and

$$
A_{2}^{*}=\operatorname{span}\left\{\left(\chi_{2}, a \chi_{2}\right)\right\}
$$

and

$$
A_{4}^{*}=\operatorname{span}\left\{\left(\chi_{4}, b \chi_{4}\right)\right\}
$$

with $a, b$ non-zero. Then $A_{2}^{*}$ and $A_{4}^{*}$ do not contain any odd vectors. Using Lemma 5.7 one computes that with $\chi=\left(\chi_{2}, a \chi_{2}\right)+\left(\chi_{4},-a^{-1} \chi_{4}\right)$,

$$
\delta\left(2 K_{J}^{*}, \chi\right)=\delta\left(K, \chi_{2}+\chi_{4}\right) \delta\left(K, a \chi_{2}-a^{-1} \chi_{4}\right) \cdot \Delta_{J}\left(\zeta^{2} t\right) \Delta_{J}\left(\zeta^{5} t\right)
$$

modulo norms, which is non-trivial by Lemma 5.6. The choice $b=-a^{-1}$ is crucial, and to establish this equality (appropriately generalized) we will have to switch to $\mathbf{Z}_{49}$ coefficients and examine the linking form more carefully. 


\subsection{Case II: Metabolizers with no odd characters}

The proof that $L$ is not slice is reduced to treating the case when $A_{2}^{*}$ and $A_{4}^{*}$ both have dimension $n / 2$ (in particular $n$ is even) and neither $A_{2}^{*}$ nor $A_{4}^{*}$ contains an odd character. We will ultimately show that in this case there nevertheless exists a $\chi \in A^{*}$ such that $\delta(L, \chi)$ is not a norm. The analysis turns out to be more complicated and, in particular, will require the use of the linking form on $M_{3}$ and $\mathbf{Z}_{49}$ coefficients.

The linking form on the 3 -fold branched cover of a knot $Z$ is a non-singular symmetric pairing

$$
l k: H_{1}\left(M_{3}(Z) ; \mathbf{Z}\right) \times H_{1}\left(M_{3}(Z) ; \mathbf{Z}\right) \rightarrow \mathbf{Q} / \mathbf{Z}
$$

Taking $Z=K$ or $Z=L$ or $Z=K_{J}^{*}$, we see that the linking form takes its values in $\mathbf{Z}_{49} \subset \mathbf{Q} / \mathbf{Z}$. Hence the adjoint of the linking form defines an isomorphism

$$
\ell: H_{1}\left(M_{3}(Z) ; \mathbf{Z}\right) \rightarrow H^{1}\left(M_{3}(Z) ; \mathbf{Z}_{49}\right)
$$

by $\ell(x)(y)=l k(x, y)$. This in turn defines a pairing

$$
l k^{*}: H^{1}\left(M_{3}(Z) ; \mathbf{Z}_{49}\right) \times H^{1}\left(M_{3}(Z) ; \mathbf{Z}_{49}\right) \rightarrow \mathbf{Z}_{49}
$$

by $l k^{*}(x, y)=l k\left(\ell^{-1}(x), \ell^{-1}(y)\right)$.

Lemma 5.12 In the basis $\chi_{18}, \chi_{30}$ for $H^{1}\left(M_{3}(K) ; \mathbf{Z}_{49}\right)$, the form $l k^{*}$ has matrix

$$
\left(\begin{array}{ll}
0 & u \\
u & 0
\end{array}\right)
$$

for some unit $u \in \mathbf{Z}_{49}$.

Proof Since $T(\ell(x))=\ell\left(T^{-1}(x)\right)$ for $x \in H_{1}\left(M_{3}(K) ; \mathbf{Z}\right)$, it follows that $T$ takes 18 eigenvectors to 30 eigenvectors and vice-versa. Hence $l k^{*}\left(\chi_{18}, \chi_{18}\right)=$ $18^{-2} l k^{*}\left(\chi_{18}, \chi_{18}\right)$ and so $l k^{*}\left(\chi_{18}, \chi_{18}\right)=0$ since $18^{-2}-1=17=26^{-1}$. Similarly $l k^{*}\left(\chi_{18}, \chi_{18}\right)=0$. The off-diagonal entries are equal since the form is symmetric, and $u$ must be a unit since the form is non-singular.

To an invariant metabolizer $A \subset H_{1}\left(M_{3}(L) ; \mathbf{Z}\right) \cong\left(\mathbf{Z}_{49}\right)^{2 n}$ we have associated the group $A^{*} \subset H^{1}\left(M_{3}(L) ; \mathbf{Z}_{7}\right) \cong\left(\mathbf{Z}_{7}\right)^{2 n}$ of $\mathbf{Z}_{7}$ characters which vanish on $A$.

Lemma 5.13 Let $A \subset\left(\mathbf{Z}_{49}\right)^{2 n}$ be an invariant metabolizer. Suppose that $A^{*}$ is isomorphic to $\left(\mathbf{Z}_{7}\right)^{n}$. Suppose that $a_{1} \cdots, a_{n}$ and $b_{1}, \cdots, b_{n}$ are integers so that $\left(a_{1} \chi_{2}, \cdots, a_{n} \chi_{2}\right) \in A_{2}^{*}$ and $\left(b_{1} \chi_{4}, \cdots, b_{n} \chi_{4}\right) \in A_{4}^{*}$. Then $\sum_{i} \epsilon_{i} a_{i} b_{i}=0$ $(\bmod 7)$. 
Proof Write $M$ for $M_{3}(L)$. We always identify the group $H^{1}\left(M ; \mathbf{Z}_{7}\right)$ with $7 H^{1}\left(M ; \mathbf{Z}_{49}\right) \subset H^{1}\left(M ; \mathbf{Z}_{49}\right)$. Recall that $\chi_{2}=7 \chi_{30}$ and $\chi_{4}=7 \chi_{18}$ and therefore we have

$$
\left(a_{1} \chi_{2}, \cdots, a_{n} \chi_{2}\right)=7\left(a_{1} \chi_{30}, \cdots, a_{n} \chi_{30}\right)
$$

and

$$
\left(b_{1} \chi_{4}, \cdots, b_{n} \chi_{4}\right)=7\left(b_{1} \chi_{18}, \cdots, b_{n} \chi_{18}\right) .
$$

Since $A$ is a subgroup of $\left(\mathbf{Z}_{49}\right)^{2 n}, A \cong \mathbf{Z}_{49}^{a} \oplus \mathbf{Z}_{7}^{b}$. Since $A$ is a metabolizer, its order is $49^{n}$ and so $2 a+b=2 n$. The group $A^{*}$ equals $\operatorname{Hom}\left(H_{1}(M) / A ; \mathbf{Z}_{7}\right)$. But $H_{1}(M) / A \cong A$. To see this, note that the sequence

$$
0 \rightarrow A \rightarrow H_{1}(M) \stackrel{\ell}{\longrightarrow} \operatorname{Hom}(A ; \mathbf{Q} / \mathbf{Z}) \rightarrow 0
$$

is exact, where $\ell(x)(y)=l k(x, y)$, since the linking form is non-degenerate. But since $A$ is a finite abelian group, $\operatorname{Hom}(A ; \mathbf{Q} / \mathbf{Z})$ is isomorphic to $A$.

Thus $\left(\mathbf{Z}_{7}\right)^{n} \cong A^{*} \cong \operatorname{Hom}\left(A, \mathbf{Z}_{7}\right) \cong\left(\mathbf{Z}_{7}\right)^{a+b}$, so $n=a+b$. Together with $2 a+b=2 n$ this implies that $a=n$ and $b=0$, so $A \cong\left(\mathbf{Z}_{49}\right)^{n}$.

Now let $\tilde{A} \subset H^{1}\left(M ; \mathbf{Z}_{49}\right)$ denote the set of $\mathbf{Z}_{49}$ characters which vanish on $A$; the map $\ell$ takes $A$ isomorphically to $\tilde{A}$, so that $\tilde{A} \cong\left(\mathbf{Z}_{49}\right)^{n}$. It follows easily that $A^{*}=7 \tilde{A}$.

Therefore, there exist $x, y \in H^{1}\left(M ; \mathbf{Z}_{7}\right)$ so that

$$
\left(a_{1} \chi_{30}, \cdots, a_{n} \chi_{30}\right)+7 x
$$

and

$$
\left(b_{1} \chi_{18}, \cdots, b_{n} \chi_{18}\right)+7 y
$$

are both in $\tilde{A}$. Since $M$ is a connected sum, the linking form $l k^{*}$ splits according to the splitting of $H^{1}\left(M ; \mathbf{Z}_{49}\right)=\sum_{i=1}^{n} H^{1}\left(M_{3}\left(\epsilon_{i} K_{J_{i}}^{*}\right) ; \mathbf{Z}_{29}\right)$. On each summand, the linking matrix is

$$
\epsilon_{i}\left(\begin{array}{ll}
0 & u \\
u & 0
\end{array}\right)
$$

using Lemma 5.12 and the fact that $M_{3}(-Z)=-M_{3}(Z)$.

Thus

$$
\begin{gathered}
0=l k\left(\left(a_{1} \chi_{30}, \cdots, a_{n} \chi_{30}\right)+7 x,\left(b_{1} \chi_{18}, \cdots, b_{n} \chi_{18}\right)+7 y\right) \\
=u \sum_{i} \epsilon_{i} a_{i} b_{i} \quad(\bmod 7) .
\end{gathered}
$$

We can now return to the argument that $L$ is not slice. We have treated all cases except when $n=2 k, A_{2}^{*}$ and $A_{4}^{*}$ are both $k$ dimensional and contain no odd eigencharacters. 
A vector in $A_{2}^{*}$ can be expressed as an $n$-tuple in $\mathbf{Z}_{7}$ using the correspondence

$$
\left(a_{1}, \cdots, a_{n}\right) \leftrightarrow\left(a_{1} \chi_{2}, \cdots a_{n} \chi_{2}\right) .
$$

This way any basis for $A_{2}^{*}$ determines a $k \times 2 k$ matrix $(D E)$ whose rows are the basis vectors. Similarly a basis for $A_{4}^{*}$ determines a $k \times 2 k$ matrix $(F G)$.

As remarked after Lemma 5.11, if the matrix $(D E)$ can be reduced to $(I B)$ with $B$ singular by performing elementary row operations and column interchanges, then $A_{2}^{*}$ contains an odd character $\chi$ and so by Corollary 5.10 for this odd character $\delta(L ; \chi)$ is not a norm. Thus it remains only to treat the case when in any such reduction the matrix $B$ is non-singular, and similarly for the 4-eigenspace $A_{4}^{*}$.

Lemma 5.13 above implies that

$$
\left(\begin{array}{ll}
D & E
\end{array}\right) S\left(\begin{array}{l}
F^{t} \\
G^{t}
\end{array}\right)=0
$$

where $S$ is the diagonal matrix with diagonal entries the signs $\epsilon_{1}, \cdots, \epsilon_{n}$.

There exists an invertible matrix $K \in G L_{k}(\mathbf{Z})$ and a $2 k \times 2 k$ permutation matrix $P$ so that $K(D E) P^{-1}=(I B)$, since elementary row operations and column interchanges reduce the rank $k$ matrix $(D E)$ to $(I B)$ with $B$ nonsingular. Changing the basis of $A_{2}^{*}$ we may assume that $K=I$, so that $(D E)=\left(\begin{array}{ll}I & B\end{array}\right) P$.

Define $k \times k$ matrices $X$ and $Y$ by $(X Y)=(F G) P^{-1}$, and define two $k \times k$ diagonal matrices $S_{1}$ and $S_{2}$ with diagonal entries the signs $\epsilon_{i}$ permuted by $P$ by

$$
\left(\begin{array}{cc}
S_{1} & 0 \\
0 & S_{2}
\end{array}\right)=P S P^{-1}
$$

Then

$$
\begin{aligned}
0 & =\left(\begin{array}{ll}
D & E
\end{array}\right) S\left(\begin{array}{c}
F^{t} \\
G^{t}
\end{array}\right) \\
& =\left(\begin{array}{ll}
I & B
\end{array}\right)\left(\begin{array}{cc}
S_{1} & 0 \\
0 & S_{2}
\end{array}\right)\left(\begin{array}{l}
X^{t} \\
Y^{t}
\end{array}\right) \\
& =S_{1} X^{t}+B S_{2} Y^{t}
\end{aligned}
$$

Hence $Y=-X S_{1}\left(B^{t}\right)^{-1} S_{2}^{-1}$ and so

$$
(F G)=(X Y) P=X\left(I-S_{1}\left(B^{t}\right)^{-1} S_{2}\right) P .
$$

The matrix $X$ must therefore be invertible and so by changing basis of $A_{4}^{*}$ by the matrix $X S_{1}$ we can assume $(F G)=\left(I-\left(B^{t}\right)^{-1}\right) P S$. 
By assumption $A_{2}^{*}$ and $A_{4}^{*}$ contain no odd vectors. Thus we are poised to apply the following lemma, the proof of which is given later in this section.

Lemma 5.14 Let $E$ be a non-singular $k \times k$ matrix over $\mathbf{Z}_{p}$ for a prime $p>2$. Suppose that the subspace of $\left(\mathbf{Z}_{p}\right)^{2 k}$ spanned by the rows of the $k \times 2 k$ matrix $(I E)$ contains no odd vectors (ie, every vector in this span has an even number of non-zero entries). Then $E$ is obtained from a diagonal matrix by permuting the columns.

The proof that $L$ is not slice is completed as follows. Lemma 5.14 implies that the matrix $B$ is obtained from a diagonal matrix by permuting the columns, say $B=C Q$ for a diagonal matrix $C=\operatorname{diag}\left(c_{1}, \ldots, c_{n}\right)$ and a $k \times k$ permutation matrix $Q$. Write $R=\left(\begin{array}{cc}I & 0 \\ 0 & Q\end{array}\right) P$; thus $R$ is a $2 k \times 2 k$ permutation matrix and

$$
(D E)=(I C) R, \quad(F G)=\left(I-C^{-1}\right) R S .
$$

By reordering the summands of $L=\epsilon_{1} K_{J_{1}}^{*} \# \cdots \# \epsilon_{n} K_{J_{n}}^{*}$ we may assume that $R$ is the identity permutation. Thus $A_{2}^{*}$ contains the character

$$
\alpha_{2}=\left(\chi_{2}, 0, \ldots, 0, c_{1} \chi_{2}, 0, \ldots, 0\right)
$$

and $A_{4}^{*}$ contains the character

$$
\alpha_{4}=\left(\epsilon_{1} \chi_{4}, 0, \ldots, 0,-\epsilon_{k+1} c_{1}^{-1} \chi_{4}, 0, \ldots, 0\right)
$$

where for each vector the second non-zero entry is in the $k+1$ st position. Thus any linear combination of these characters lies in $A^{*}=A_{2}^{*} \oplus A_{4}^{*}$.

Let $\chi=\alpha_{2}+\epsilon_{1} \alpha_{4}$. We claim that $\delta(L, \chi)$ is not a norm.

For ease of notation write $J=J_{1}$ and $J^{\prime}=J_{k+1}, \epsilon=\epsilon_{1} \epsilon_{k+1}, c=c_{1}$, and $\bar{c}=c^{-1}(\bmod 7)$.

Lemmas 5.7 and 5.6 show that, modulo norms, $\delta(L, \chi)$ is the product of the four terms:

(1) $\delta\left(K, \chi_{2}+\epsilon \chi_{4}\right)$,

(2) $\delta\left(K, c \chi_{2}-\epsilon^{\prime} c^{-1} \chi_{4}\right)$

(3) $\Delta_{J}\left(\zeta^{2} t\right) \Delta_{J}\left(\zeta^{6} t\right) \Delta_{J}\left(\zeta^{6} t\right) \Delta_{J}\left(\zeta^{5} t\right) \Delta_{J}(\zeta t) \Delta_{J}(\zeta t) \equiv \Delta_{J}\left(\zeta^{2} t\right) \Delta_{J}\left(\zeta^{5} t\right)(\bmod -$ ulo norms)

(4) $\Delta_{J^{\prime}}\left(\zeta^{c-\epsilon \bar{c}} t\right) \Delta_{J^{\prime}}\left(\zeta^{2 c-4 \epsilon \bar{c}} t\right) \Delta_{J^{\prime}}\left(\zeta^{4 c-2 \epsilon \bar{c}} t\right)$.

$$
\Delta_{J^{\prime}}\left(\zeta^{-c+\epsilon \bar{c}} t\right) \Delta_{J^{\prime}}\left(\zeta^{-2 c+4 \epsilon \bar{c}} t\right) \Delta_{J^{\prime}}\left(\zeta^{-4 c+2 \epsilon \bar{c}} t\right) .
$$


There are two cases to consider, depending on whether or not $J=J^{\prime}$. If $J=J^{\prime}$, ie, $J_{1} \neq J_{k+1}$, then $\delta(L, \chi)$ is not a square since the third term above is not a norm and cannot cancel with any of the other three terms by the choice of the $S_{r}$.

If $J_{1}=J_{k+1}$, then $\epsilon_{1}=\epsilon_{k+1}$, so that $\epsilon=1$. Then the exponents of $\zeta$ in the fourth term above are

$$
\{c-\bar{c}, 2 c-4 \bar{c}, 4 c-2 \bar{c},-c+\bar{c},-2 c+4 \bar{c},-4 c+2 \bar{c}\} .
$$

The reader can check that for each non-zero choice of $c$ in $\mathbf{Z}_{7}$, this (unordered) set is just $\{0,0,2,2,5,5\}$, so that the fourth term is always a norm. Since the third term is not a norm, the product of the third and fourth terms is not a norm, and so $\delta(L, \chi)$ is not a norm.

This completes the argument that $L$ is not slice, since for each invariant metabolizer $A$ we have shown how to construct a character $\chi \in A^{*}$ for which $\delta(K, \chi)$ is not a norm. Hence Theorem 5.3 is proven.

\subsection{The proof of Lemma 5.14}

Proof Consider the columns of $E$ as a basis $e_{i}$ of $V=\left(\mathbf{Z}_{p}\right)^{k}$. Let $b_{1}, \cdots, b_{n}$ denote the standard basis of $V$. Let $V^{*}=\operatorname{Hom}\left(V, \mathbf{Z}_{p}\right)$. The condition that every linear combination of the rows of $(I E)$ is even can be restated by saying that for each $x \in V^{*}$, the sets

$$
\left\{j \mid x\left(e_{j}\right) \neq 0\right\} \text { and }\left\{j \mid x\left(b_{j}\right) \neq 0\right\}
$$

have the same cardinality modulo 2 .

Another way to state this is as follows. Let $h_{j}: V^{*} \rightarrow \mathbf{C}$ be the function

$$
h_{j}(x)=\left\{\begin{aligned}
1 & \text { if } x\left(e_{j}\right)=0 \\
-1 & \text { if } x\left(e_{j}\right) \neq 0
\end{aligned}\right.
$$

and let $H: V^{*} \rightarrow \mathbf{C}$ be the product $H(x)=\prod_{j=1}^{k} h_{j}(x)$. Similarly define $g_{j}: V^{*} \rightarrow \mathbf{C}$ by

$$
g_{j}(x)=\left\{\begin{aligned}
1 & \text { if } x\left(b_{j}\right)=0 \\
-1 & \text { if } x\left(b_{j}\right) \neq 0
\end{aligned}\right.
$$

and $G: V^{*} \rightarrow \mathbf{C}$ be the product $G(x)=\prod_{j=1}^{k} g_{j}(x)$. Then the hypothesis is equivalent to the statement that $H=G$. 
Let $\hat{H}: V \rightarrow \mathbf{C}$ be the Fourier transform

$$
\hat{H}(v)=\sum_{x \in V^{*}} H(x) e^{\frac{2 \pi i}{p} x(v)} .
$$

Define $\hat{G}: V \rightarrow \mathbf{C}$ similarly using $G$.

We compute $\hat{H}$ as follows. Let $\left\{e_{j}^{*}\right\} \subset V^{*}$ denote the dual basis to the $e_{j}$. Then

$$
\hat{H}(v)=\sum_{x_{1} \in \mathbf{Z}_{p}} \cdots \sum_{x_{k} \in \mathbf{Z}_{p}} H\left(\Sigma_{i} x_{i} e_{i}^{*}\right) e^{\frac{2 \pi i}{p} \Sigma_{i} x_{i} e_{i}^{*}(v)} .
$$

From its definition one sees that $h_{j}\left(\sum_{i} x_{i} e_{i}^{*}\right)=h_{j}\left(x_{j} e_{j}^{*}\right)$, so that

$$
H\left(\Sigma_{i} x_{i} e_{i}^{*}\right)=\prod_{j} h_{j}\left(x_{j} e_{j}^{*}\right) .
$$

Substituting this into Equation (3) and simplifying one gets

$$
\hat{H}(v)=\prod_{j}\left(\sum_{x \in \mathbf{Z}_{p}} h_{j}\left(x e_{j}^{*}\right) e^{\frac{2 \pi i}{p} x e_{j}^{*}(v)}\right) .
$$

Now

$$
\sum_{x \in \mathbf{Z}_{p}} h_{j}\left(x e_{j}^{*}\right) e^{\frac{2 \pi i}{p} x e_{j}^{*}(v)}=1-\sum_{x \in \mathbf{Z}_{p}-0} e^{\frac{2 \pi i}{p} x e_{j}^{*}(v)} .
$$

Notice that

$$
\sum_{x \in \mathbf{Z}_{p}-0} e^{\frac{2 \pi i}{p} x e_{j}^{*}(v)}= \begin{cases}p-1 & \text { if } e_{j}^{*}(v)=0 \\ -1 & \text { if } e_{j}^{*}(v) \neq 0 .\end{cases}
$$

Thus for each $v, \hat{H}(v)=2^{\alpha(v)}(2-p)^{k-\alpha(v)}$ where $\alpha(v)$ is the cardinality of the set $\left\{j \mid e_{j}^{*}(v) \neq 0\right\}$. Similarly $\hat{G}(v)=2^{\beta(v)}(2-p)^{k-\beta(v)}$ where $\beta(v)$ is the cardinality of the set $\left\{j \mid b_{j}^{*}(v) \neq 0\right\}$. Since $H=G, \hat{H}=\hat{G}$, and since $p>2$,

$$
2^{\alpha(v)-\beta(v)}=(2-p)^{\alpha(v)-\beta(v)} .
$$

This implies that $\alpha(v)=\beta(v)$ since $p \neq 4$ (recall that $p$ is prime). Now $\alpha\left(e_{j}\right)=1$ and therefore $\beta\left(e_{j}\right)=1$. Hence for each $j$ there exists a unique $k_{j}$ so that $b_{k_{j}}^{*}\left(e_{j}\right) \neq 0$. Since $E$ is non-singular it follows that $j \mapsto k_{j}$ is a permutation, and that $e_{j}=\left(b_{k_{j}}^{*}\left(e_{j}\right)\right) b_{k_{j}}$. In other words, $E$ is obtained from a diagonal matrix by permuting the columns. 


\section{References}

[1] S Akbulut, R Kirby, Branched covers of surfaces in 4-manifolds, Math. Ann. 252 (1979/80) 111-131

[2] M F Atiyah, V K Patodi, I M Singer, Spectral asymmetry and Riemannian Geometry. II, Math. Proc. Camb. Phil. Soc. 78 (1975) 405-432

[3] G Bredon, Introduction to compact transformation groups, Pure and Applied Mathematics, Vol. 46. Academic Press, New York-London, 1972

[4] G Burde, H Zieschang, Knots, de Gruyter Studies in Mathematics, 5, Walter de Gruyter \& Co., Berlin-New York, 1985

[5] A Casson, C McA Gordon, Cobordism of classical knots, in A la recherche de la Topologie perdue, ed. by Guillou and Marin, Progress in Mathematics, Volume 62, 1986 (Originally published as Orsay Preprint, 1975.)

[6] A Casson, C McA Gordon, On slice knots in dimension three, in Proc. Symp. Pure Math. 32 (1978) 39-54

[7] J C Cha, K H Ko, On equivariant slice knots, Proc. Amer. Math. Soc. 127 (1999) no. 7, 2175-2182

[8] T Cochran, R Gompf, Applications of Donaldson's theorems to classical knot concordance, homology 3-spheres and property P, Topology 27 (1988) 495-51

[9] T Cochran, K Orr, $\mathbf{P}$ Teichner, Knot concordance, Whitney towers, and $L^{2}$-signatures, preprint, 1999

[10] P E Conner, E E Floyd, Differentiable periodic maps, Ergebnisse der Mathematik und ihrer Grenzgebiete, N. F., Band 33 Academic Press Inc. New York; Springer-Verlag, Berlin-Göttingen-Heidelberg 1964

[11] D Coray, F Michel, Knot cobordism and amphicheirality, Comm. Math. Helv., 58 (1983) 601-616

[12] S Donaldson, An application of gauge theory to four-dimensional topology, J. Differential Geom. 18 (1983) 279-315

[13] R Fox, A quick trip through knot theory, Topology of 3-manifolds and related topics, Proc. The Univ. of Georgia Institute (1961) Prentice-Hall, Englewood Cliffs, N.J., 1962, pp. 120-167

[14] R Fox, J Milnor, Singularities of 2-spheres in 4-space and cobordism of knots, Osaka J. Math. 3 (1966) 257-267

[15] M Freedman, The topology of four-dimensional manifolds, J. Differential Geom. 17 (1982) 357-453

[16] M Freedman, F Quinn, Topology of 4-manifolds, Princeton Mathematical Series, 39 Princeton University Press, Princeton, NJ, 1990

[17] P Gilmer, Slice knots in $S^{3}$, Quart. J. Math. Oxford Ser. (2) 34 (1983) no. 135, 305-322 
[18] P Gilmer, C Livingston, The Casson-Gordon invariant and link concordance, Topology 31 (1992) no. 3, 475-492

[19] P Gilmer, C Livingston, Discriminants of Casson-Gordon invariants, Math. Proc. Camb. Phil. Soc. 112 (1992) 127-139

[20] C McA Gordon, Problems in Knot Theory, Proceedings of a Seminar held in Plans-sur-Bex, 1977, edited by Jean-Claude Hausmann. Lecture Notes in Mathematics, 685. Springer-Verlag, Berlin-New York, 1978

[21] C Herald, Flat connections, the Alexander invariant, and Casson's invariant, Comm. Anal. Geom. 5 (1997) no. 1, 93-120

[22] C Herald, Existence of irreducible representations for knot complements with nonconstant equivariant signature, Math. Ann. 309 (1997) no. 1, 21-35

[23] M Heusener, J. Kroll, Deforming abelian SU(2)-representations of knot groups, Comment. Math. Helv. 73 (1998) 480-498

[24] B Jiang, A simple proof that the concordance group of algebraically slice knots is infinitely generated, Proc. Amer. Math. Soc. 83 (1981) 189-192

[25] A Kawauchi, Topological imitation, mutation and the quantum SU(2) invariants, J. Knot Theory Ramifications 3 (1994) no. 1, 25-39

[26] C Kearton, Mutation of knots, Proc. Amer. Math. Soc. 105 (1989) no. 1, 206208

[27] M Kervaire, Knot cobordism in codimension two, Manifolds-Amsterdam 1970 (Proc. Nuffic Summer School) pp. 83-105 Lecture Notes in Mathematics, Vol. 197 Springer, Berlin (1971)

[28] R Kirby, Problems in low dimensional manifold theory, Algebraic and Geometric Topology (Stanford, 1976) vol 32, part II, Proc. Sympos. Pure Math., 273-312

[29] R Kirby, Problems in low dimensional manifold theory, Geometric Topology, AMS/IP Studies in Advanced Mathematics, ed. W. Kazez, 1997

[30] R Kirby, W B R Lickorish, Prime knots and concordance, Math. Proc. Cambridge Philos. Soc. 86 (1979) no. 3, 437-441

[31] P Kirk, C Livingston, Twisted knot polynomials: inversion, mutation and concordance, Topology 38 (1999) no. 3, 663-671

[32] P Kirk, C Livingston, Twisted Alexander invariants, Reidemeister torsion, and Casson-Gordon invariants, Topology 38 (1999) no. 3, 635-661

[33] J Levine, Knot cobordism groups in codimension two, Comment. Math. Helv. 44 (1969) 229-244

[34] J Levine, Invariants of knot cobordism, Invent. Math. 8 (1969) 98-110

[35] W B R Lickorish, Distinct 3-manifolds with all $\mathrm{SU}(2)_{q}$ invariants the same, Proc. Amer. Math. Soc. 117 (1993) no. 1, 285-292 
[36] R Litherland, A formula for the Casson-Gordon invariant of a knot, preprint (1980)

[37] R Litherland, Cobordism of Satellite Knots, Four-Manifold Theory, Contemporary Mathematics, eds. C. Gordon and R. Kirby, American Mathematical Society, Providence RI 1984, 327-362

[38] C Livingston, Homology cobordisms of 3-manifolds, knot concordances, and prime knots, Pacific J. Math. 94 (1981) no. 1, 193-206

[39] C Livingston, Knots which are not concordant to their reverses, Oxford Q. J. of Math. 34 (1983) 323-328

[40] C Livingston, Order 2 algebraically slice knots, Proceedings of the Kirbyfest, Geometry and Topology Monographs vol. 2 (1998) ed. Joel Hass and Martin Scharlemann

[41] C Livingston, S Naik, Obstructing 4-torsion in the classical knot concordance group, J. Differential Geometry 51 (1999) 1-12

[42] R Meyerhoff, D Ruberman, Mutation and the $\eta$-invariant, J. Differential Geom. 31 (1990) no. 1, 101-130

[43] R Myers, Homology cobordisms, link concordances, and hyperbolic 3-manifolds, Trans. Amer. Math. Soc. 278 (1983) no. 1, 271-288

[44] H R Morton, P Traczyk, The Jones polynomial of satellite links around mutants, Braids (Santa Cruz, CA, 1986) 587-592, Contemp. Math., 78, Amer. Math. Soc., Providence, RI, 1988

[45] D Mullins, The generalized Casson invariant for 2 -fold branched covers of $S^{3}$ and the Jones polynomial, Topology 32 (1993) no. 2, 419-438

[46] K Murasugi, On a certain numerical invariant of link types, Trans. Amer. Math. Soc. 117 (1965) 387-422

[47] K Murasugi, Knot Theory and its Application, Birkhauser, Boston, 1996

[48] S Naik, Casson-Gordon invariants of genus one knots and concordance to reverses, J. of Knot Theory and its Ramifications 5 (1996) 661-677

[49] S Naik, Equivariant concordance of knots in $S^{3}$, KNOTS '96 (Tokyo), 81-89, World Sci. Publishing, River Edge, NJ, 1997

[50] J Porti, Torsion de Reidemeister pour les variétés hyperboliques, Mem. Amer. Math. Soc. 128 (1997) no. 612

[51] D Rolfsen, Knots and Links, Publish or Perish, Berkeley CA (1976)

[52] Y W Rong, Mutation and Witten invariant, Topology 33 (1994) no. 3, 499-507

[53] D Ruberman, Mutation and volumes of knots in $S^{3}$, Invent. Math. 90 (1987) no. 1, 189-215.

[54] L Rudolph, An obstruction to sliceness via contact geometry and "classical" gauge theory, Invent. Math. 119 (1995) 155-163 
[55] J P Serre, Linear Representations of Finite Groups, Graduate Texts in Mathematics, No. 42. Springer-Veralg, New York, 1977

[56] A Tristram, Some cobordism invariants for links, Proc. Camb. Phil. Soc. 66 (1969) 251-264

[57] H Trotter, On S-equivalence of Seifert matrices, Invent. Math. 20 (1973) 173207

[58] H Trotter, Non-invertible knots exist, Topology 2 (1963) 275-280

[59] K Walker, An extension of Casson's invariant, Annals of Mathematics Studies, 126. Princeton University Press, Princeton, NJ, 1992

[60] C T C Wall, Non-additivity of the signature, Invent. Math. 7 (1969) 269-274

[61] E Witten, Monopoles and four-manifolds, Math. Res. Lett. 1 (1994) no. 6, 769-796 